\title{
A Novel Distance Measure for Pythagorean Fuzzy Sets and its Applications to the Technique for Order Preference by Similarity to Ideal Solutions
}

\author{
Fang Zhou ${ }^{1}$, Ting-Yu Chen ${ }^{2,3,4, *}$ \\ ${ }^{1}$ Graduate Institute of Business and Management, Chang Gung University, Department of Economic and Management, Suzhou Vocational Institute of Industrial \\ Technology, No.1, Zhineng Avenue, Suzhou City, 215104, China \\ ${ }^{2}$ Graduate Institute of Business and Management, Chang Gung University, No. 259, Wenhua 1st Rd., Guishan District, Taoyuan City 33302, Taiwan \\ ${ }^{3}$ Department of Industrial and Business Management, Chang Gung University, No. 259, Wenhua 1st Rd., Guishan District, Taoyuan City 33302, Taiwan \\ ${ }^{4}$ Department of Nursing, Linkou Chang Gung Memorial Hospital, No. 259, Wenhua 1st Rd., Guishan District, Taoyuan City 33302, Taiwan
}

ARTICLE IN F O
Article History
Received 25 May 2019
Accepted 19 Aug 2019
Keywords
Pythagorean fuzzy set
Multicriteria decision-making
(MCDM)
Distance measure
Technique for order preference by
similarity to ideal solutions
(TOPSIS)

\section{ABSTRACT}

Ever since the introduction of Pythagorean fuzzy (PF) sets, many scholars have focused on solving multicriteria decision-making (MCDM) problems with PF information. The technique for order preference by similarity to ideal solutions (TOPSIS) is a wellknown and effective method for MCDM problems. The objective of this study is to extend the TOPSIS to tackle MCDM problems under the PF environment. In this study, we develop a novel distance measure that considers the length, the angle, and the greater space, which reflect the properties of PF sets. Then, we apply the proposed distance measure in PF-TOPSIS to calculate the distances from the PF positive ideal solution and the PF negative ideal solution. Finally, we take the evaluation of emerging technology commercialization as an MCDM problem to illustrate the proposed approaches, and we then compare these approaches to demonstrate the scalar type PF-TOPSIS is the most feasible and effective approach in practice.

(C) 2019 The Authors. Published by Atlantis Press SARL. This is an open access article distributed under the CC BY-NC 4.0 license (http://creativecommons.org/licenses/by-nc/4.0/).

\section{INTRODUCTION}

Multicriteria decision-making (MCDM) refers to the identification of the optimal alternative or the determination of the ranking order of all alternatives. Due to the inherent vagueness of decision makers' preferences in practice, Zadeh [1] introduced the concept of fuzzy sets for dealing with MCDM problems, which use the membership degree to denote the alternative satisfies the criterion. However, in practice, a decision maker usually points out the degree to which the alternative fails to satisfy the criterion. Atanassov [2] initially developed the intuitionistic fuzzy (IF) sets, which utilize the membership degree and the nonmembership degree and allow the sum of the two degrees to be equal to or less than one. However, we may encounter the problem that the sum of the two degrees is greater than one in a real decision process. To overcome this restriction, Yager [3,4] introduced the Pythagorean fuzzy (PF) sets, with the condition that the sum of the squares of the membership degree and the nonmembership degree is equal to or less than one. PF sets outperform IF sets and can deal with more ambiguous and uncertain information in MCDM practice.

Ever since the introduction of PF sets, many scholars have focused on solving MCDM problems under the PF environment. Zhang and

*Corresponding author. Email: tychen@mail.cgu.edu.tw
$\mathrm{Xu}$ [5], Zeng et al. [6], Gary [7], Biswas and Sarkar [8], Yu et al. [9], and Akram et al. [10] extended the technique for order preference by similarity to ideal solutions (TOPSIS) method with PF information to solve MCDM problems. Ren et al. [11] and Biswas and Sarkar [12] proposed the PF-TODIM (acronym in Portuguese for interactive MCDM) approach for MCDM problems. Zhang [13] developed the Pythagorean fuzzy qualitative flexible multiple criteria method (PF-QUALIFLEX) for dealing with hierarchical MCDM problems. Chen [14], Gul et al. [15], and Liang et al. [16] proposed the PF-Vise Kriterijumska Optimizacija I Kompromisno Resenje (VIKOR) method for dealing with MCDA problems. Wang and Chen [17] developed an effective assignment-based method using correlation-based precedence indices for MCDM problems within the PF uncertain environment. Haktanir and Kahraman [18] proposed the interval-valued Pythagorean fuzzy quality function development (IVPF-QFD) method for handling solar photovoltaic technology problems. Liu et al. [19] integrated QFD and QUALIFLEX to solve the robot selection problem under the PF environment. Yang et al. [20] developed an IVPF Frank power weighted average operator-based technique to deal with multiple attribute group decision-making problems.

TOPSIS [21] is a well-known and widely accepted method for MCDM problems, which follows the strategy of selecting the solution that has the shortest distance from the positive ideal 
solution (PIS) and the farthest distance from the negative ideal solution (NIS). Because TOPSIS is a simple yet effective approach for MCDM problems, researchers have applied TOPSIS for MCDM problems within the $\mathrm{PF}$ environment. Zhang and $\mathrm{Xu}[5]$ extended TOPSIS with PF information based on score function and a scorebased ranking. Zeng et al. [6] introduced the PF ordered weighted averaging weighted average distance into TOPSIS by integrating the subjective information and the attitudinal characteristics of decision makers' preferences. Gary [7] introduced an improved score function into TOPSIS with IVPF information. Wan et al. [22] developed a novel ranking method according to the arc-length-based relative closeness (RC) degree, which was inspired by the strategy of TOPSIS. Onar et al. [8] used PF-TOPSIS to evaluate cloud service providers. Yu et al. [9] extended the TOPSIS method via the integration of the distance and the similarity to evaluate suppliers' performance using IVPF information. Akram et al. [10] extended TOPSIS to address group decision-making problems in the PF scenario.

The distance measure is essential in PF-TOPSIS due to the fundamental TOPSIS strategy of considering the distances to both the Pythagorean fuzzy positive ideal solution (PFPIS) and the Pythagorean fuzzy negative ideal solution (PFNIS). Distance measure between fuzzy sets is an important means to quantify the degree of difference in fuzzy mathematics. A large number of scholars have presented various distance measurement formulas from different viewpoints [23-27]. Li et al. [26,27] proposed the divergence measure based on dissimilarity function and fuzzy equivalence. Szmidt and Kacprzyk [28] considered three parameters that reflects the properties of IF sets and extended distance measures to IF environments. Grzegorzewsk [29] developed the distance measure for IF sets based on the Hausdorff metric. Montes et al. [30] defined the divergence to measure the distance between two IF sets. He et al. [31] defined the IF dissimilarity function based on quaternary functions.

Ever since the appearance of the PF sets, some researchers have extended the distance measure of IF sets to PF sets. Zhang and $\mathrm{Xu}$ [5] considered three parameters of PFSs, namely, the membership degree, the nonmembership degree, and the hesitation degree, while ignoring the direction of commitment, the strength of commitment, and the radian. Li and Zeng [32] considered four basic parameters (the membership degree, the nonmembership degree, the strength of commitment, and the direction of commitment) of $\mathrm{PF}$ sets in the distance measure equation. Zeng et al. [33] incorporated a parameter, namely, the hesitation degree, into Li and Zeng's method [32]; both approaches ignore the angle and the procedure is directly extended from the IF sets but does not consider the greater space of the PF sets. Wang et al. [34] introduced a distance measure that is based on the length distance and the angular distance in a bidirectional projection model under the PF environment. $\mathrm{Yu}$ et al. [35] proposed a new distance formula that employs induced ordered weighted averaging (IOWA) with PF information; however, this basic distance formula considers only three parameters, which are the same as the parameters that are considered in the method of Zhang and $\mathrm{Xu}$ [5]. Peng and Li [36] proposed a new distance measure for IVPF sets that has two parameters (the membership degree and the nonmembership degree) for resolving the counterintuitive situation.

Although scholars have focused on distance measures for PF sets, two main difficulties remain: failure to consider the unique parameters and maintenance of the greater space of PF sets. Zhang and $\mathrm{Xu}$ [5] considered the greater space of PF sets utilizing the sum of squared deviations of the membership degree, nonmembership degree, and hesitation degree, but they ignored the strength of commitment, the direction of commitment, and the radian. This limitation resulted in failure to satisfy the fundamental properties of PF sets, which obtained unreasonable results. Li and Zeng [32] and Zeng et al. [33] considered the direction of commitment and the strength of commitment, but the procedure of the distance formulas simply utilized the sum of deviations of the corresponding parameters. This difficulty resulted in failure to ensure the greater space because the maximum distance of PF sets couldn't arrive to one. Hence, the motivation of this paper is to propose a novel distance measure to overcome the difficulties of the existing distance measures for PF sets, ensure the greater space of the PF sets, consider all the related parameters that reflects the unique properties of PF sets, and satisfy the basic properties of distance measure.

The purpose of this paper is to propose a novel distance measurement method that considers the length distance, the angular distance, and the greater space, which is applied in PF-TOPSIS for MCDM problems. We first define a novel distance formula that resolves these limitations by considering the membership degree, the nonmembership degree, and the strength of commitment as the parameters for the length distance measure; by considering the direction of commitment and the radian as the parameters for the angular distance measure; and by using the square deviations of the membership degree, the nonmembership degree, and the strength of commitment, the deviation of the direction of commitment and the sin value of the angle to maintain the greater space of the PF sets. Next, we propose the PF-TOPSIS approach for MCDM problems, in which we utilize four approaches to identify the PFPIS and the PFNIS: the first is the classical type that is utilized by the union and intersection operators; the second is the fixed type that is utilized by the fixed extremum; the third is the scored type that is utilized by the score function; the fourth is the scalar type that is utilized by scalar function. Then, we use the proposed distance measurement method to calculate the distances of each alternative from the PFPIS and the PFNIS to fully reflect the properties of the PF sets and we employ both the relative closeness index and the revised closeness index to obtain the ranking order and to identify the optimal solution. Moreover, as a practical MCDM problem, this paper considers the evaluation of emerging technology commercialization to evaluate the proposed approaches and to conduct a comparative analysis among these approaches, the results of which demonstrate that the scalar type approach is the most feasible, effective, and credible approach for MCDM problems in practice. Finally, this paper proposes directions for future research.

The main contributions of this study relative to the existing distance measurement method and the existing PF-TOPSIS method are summarized as follows:

1. A novel distance measurement method for PF sets is proposed, the parameters of which reflect the length distance and angular distance based on the characteristics of PF sets and the procedure of which ensures that the greater space of PF sets is maintained. Therefore, the proposed distance measurement method measures the distance between two PF sets more accurately.

2. A scalar function is used to determine the PFPIS and the PFNIS in the PF-TOPSIS approach. Due to the unique properties of $\mathrm{PF}$ sets, the scalar function compares the magnitudes more 
accurately according to Zeng et al. [33]. However, the scalar function has yet to be applied in PF-TOPSIS. In this paper, we utilize the scalar function as an approach for comparing the magnitudes of PF sets for the determination of the PFPIS and the PFNIS, which may yield more accurate results.

3. The proposed distance measurement method is applied in PFTOPSIS to calculate the distances from the PFPIS and the PFNIS. Due to the effectiveness of the proposed distance measurement method, which is based on the properties of the PF sets, it can calculate the distances from the PFPIS and the PFNIS more precisely.

4. Substantial improvement has been realized by the PF-TOPSIS methodology in solving MCDM problems. This paper demonstrates the scalar type approach to determine the PFPIS and the PFNIS and utilizes the novel distance measurement method to calculate the distances from the PFPIS and the PFNIS, both of which reflect the properties and characteristics of PF sets, and to make the scalar type approach more effective and practicable for solving MCDM problems compared to the classical type, fixed type, and the scored type approaches.

The remainder of this paper is organized as follows: In Section 2, we briefly recall the basic concepts of PF sets, PF numbers, operations, and magnitude comparison methods. In Section 3, we compare various existing distance measurement methods and propose a novel distance measurement method that considers five parameters of PF sets. In Section 4, we propose PF-TOPSIS for solving MCDM problems with PF information. In Section 5, we consider the "evaluation of emerging technology commercialization" as an illustrative example on which to demonstrate the proposed method and conduct a comparative analysis. In Section 6, we present the conclusions of this study and discuss future work.

\section{PRELIMINARIES}

In this section, we recall basic concepts, properties, operations, and magnitude comparison methods of PF sets and PF numbers.

Definition 1. [5] Let $X$ be a set of finite universal sets. A PF set is an object that has the following form:

$$
p=\left\{<x, P\left(\mu_{p}(x), v_{p}(x)\right) \mid x \in X>\right\},
$$

where the function $\mu_{p}(x): X \rightarrow[0,1]$ denotes the membership degree of element $x \in X$ to set $p$ and the function $\nu_{p}(x): X \rightarrow[0,1]$ denotes the nonmembership degree of element $x \in X$ to set $p$. For any PF set $p$ and $x \in X$,

$$
0 \leq\left(\mu_{p}(x)\right)^{2}+\left(\nu_{p}(x)\right)^{2} \leq 1 .
$$

The function $h_{p}(x)$ denotes the hesitation degree of element $x \in X$ to set $p$. For any PF set $p$ and $x \in X$,

$$
h_{p}(x)=\sqrt{1-\left(\mu_{p}(x)\right)^{2}-\left(\nu_{p}(x)\right)^{2}} .
$$

For convenience, Zhang and $\mathrm{Xu}$ [5] defined the Pythagorean fuzzy number (PFN) as follows: A PF set $P\left(\mu_{p}(x), \nu_{p}(x)\right)$ can be expressed as a PFN $P\left(\mu_{p}, v_{p}\right)$, where $\mu_{p}, v_{p} \in[0,1], h_{p}=$ $\left(1-\left(\mu_{p}\right)^{2}-\left(\nu_{p}\right)^{2}\right)^{1 / 2}$, and $0 \leq\left(\mu_{p}\right)^{2}+\left(\nu_{p}\right)^{2} \leq 1$.
Yager [3] proposed another PFN formulation, namely, $p=$ $P\left(r_{p}, d_{p}\right)$, where $r_{p}$ defines the strength of commitment and $r_{p} \in$ $[0,1]$. The larger the value of $r_{p}$, the stronger the commitment and the lower the uncertainty of the commitment. $d_{p}$ denotes the direction of commitment. $r_{p}$ and $d_{p}$ are associated with a pair of membership grades, namely, $\mu_{p}$ and $\nu_{p}$, which correspond to the support for and against the membership; $\mu_{p}=r_{p} \cos \left(\theta_{p}\right)$ and $\nu_{p}=r_{p} \sin \left(\theta_{p}\right)$, where $\theta_{p}$ denotes the radian, which is in the range of $[0, \pi / 2]$ and defined as $\theta_{p}=\left(1-d_{p}\right)(\pi / 2)$. Alternatively, $P\left(r_{p}, d_{p}\right)$ can be expressed in polar coordinates $\left(r_{p}, \theta_{p}\right)$. The relationship between $d_{p}$ and $\theta_{p}$ can be expressed as $d_{p}=1-2\left(\theta_{p}\right) / \pi$.

The parameters of PFNs include $\mu_{p}, v_{p}, h_{p}, r_{p}, d_{p}$, and $\theta_{p}$, which are based on the concepts and properties of PFNs. From $\mu_{p}, v_{p} \in[0,1]$, it follows that $\left(\mu_{p}\right)^{2} \leq \mu_{p},\left(v_{p}\right)^{2} \leq v_{p}$; hence, a PFN has a larger membership grade range compared to an IF number.

Example 1. Let $p=P(0.8,0.3)$ be a PFN, then six parameters of the PFN $p$ are calculated in accordance with aforementioned definition as below, and the graphical representation is presented in Fig. 1. Here, $\mu_{p}=0.8, v_{p}=0.3, h_{p}=\left(1-0.8^{2}-0.3^{2}\right)^{1 / 2}=0.5196$, $r_{p}=\left(0.8^{2}+0.3^{2}\right)^{1 / 2}=0.8544, \theta_{p}=\arctan (0.3 / 0.8)=0.3588$, and $d_{p}=1-2 \times 0.3588 / \pi=0.7716$.

Definition 2. [3] Let $p_{1}=P\left(\mu_{p 1}, v_{p 1}\right), p_{2}=P\left(\mu_{p 2}, v_{p 2}\right)$, and $p=$ $P\left(\mu_{p}, v_{p}\right)$ be three PFNs. The basic operations (the union operator, the intersection operator, and the complement operator) on PFNs can be expressed as follows:

$$
\begin{array}{ll}
\text { i. } & p_{1} \cup p_{2}=P\left(\max \left\{\mu_{p 1}, \mu_{p 2}\right\}, \min \left\{v_{p 1}, v_{p 2}\right\}\right) . \\
\text { ii. } & p_{1} \cap p_{2}=P\left(\min \left\{\mu_{p 1}, \mu_{p 2}\right\}, \max \left\{v_{p 1}, v_{p 2}\right\}\right) . \\
\text { iii. } & p^{c}=P\left(v_{p}, \mu_{p}\right) .
\end{array}
$$

Zeng et al. [33] further proposed the following operation with respect to $d_{p}$ between two PFNs:

iv. $\left|d_{p 1}-d_{p 2}{ }^{c}\right|=\left|d_{p 1}{ }^{c}-d_{p 2}\right|$.

Example 2. Let $p_{1}=P(0.8,0.3), p_{2}=P(0.7,0.2)$ be two PFNs. According to Definition 2, we have:

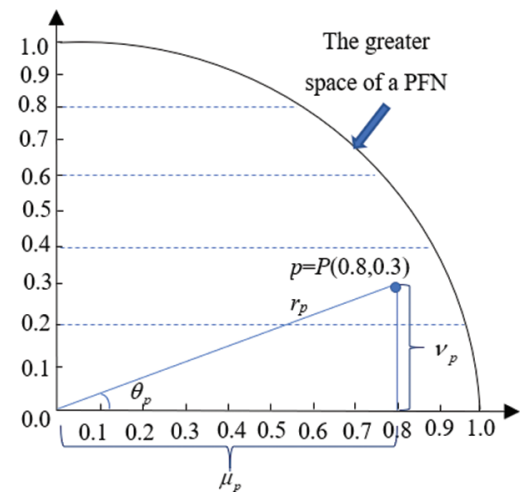

Figure 1 Graphical representation of the parameters of a Pythagorean fuzzy number (PFN). 
$p_{1} \cup p_{2}=P(\max \{0.8,0.7\}, \min \{0.3,0.2\})=P(0.8,0.2), p_{1} \cap p_{2}=$ $P(\min \{0.8,0.7\}, \max \{0.3,0.2\})=P(0.7,0.3), p_{1}^{c}=P(0.3,0.8)$, $p_{2}^{c}=P(0.2,0.7) \cdot\left|d_{p 1}-d_{p 2}{ }^{c}\right|=|0.7716-0.1772|=0.5944$, and $\left|d_{p 1}{ }^{c}-d_{p 2}\right|=|0.2284-0.8228|=0.5944$, then $\left|d_{p 1}-d_{p 2}{ }^{c}\right|=\mid d_{p 1}{ }^{c}-$ $d_{p 2} \mid$.

After the inception of PF sets and PFNs, the magnitude comparison of PFNs attracted substantial attention from scholars.

Definition 3. [5] Let $p=P\left(\mu_{p}, v_{p}\right)$ be a PFN. The score function of $p$ is defined as follows:

$$
s(p)=\left(\mu_{p}\right)^{2}-\left(v_{p}\right)^{2},
$$

where $s(p) \in[-1,1]$.

According to Peng and Yang [37], the score function was not sufficiently effective if the same values of the score function occurred in different PFNs. Then, they developed the accuracy function for improving the effectiveness of the magnitude comparison for PFNs.

Definition 4. [37] Let $p=P\left(\mu_{p}, v_{p}\right)$ be a PFN. The accuracy function of $p$ is defined as follows:

$$
a(p)=\left(\mu_{p}\right)^{2}+\left(\nu_{p}\right)^{2}
$$

where $a(p) \in[0,1]$.

Zhang [13] utilized the closeness index to compare the magnitudes between two PFNs.

Definition 5. [13] Let $p=P\left(\mu_{p}, \nu_{p}\right)$ be a PFN. The closeness index of $p$ is calculated as follows:

$$
c(p)=\frac{1-\left(\nu_{p}\right)^{2}}{2-\left(\mu_{p}\right)^{2}-\left(v_{p}\right)^{2}},
$$

where $c(p) \in[0,1]$.

Yager [3] utilized the scalar function to compare the magnitudes of PFNs, which considers parameters $r_{p}, d_{p}$, and $\theta_{p}$.

Definition 6. [3] Let $p=P\left(r_{p}, d_{p}\right)$ be a PFN. The scalar function of $p$ is defined as follows:

$$
V(p)=\frac{1}{2}+r_{p}\left(d_{p}-\frac{1}{2}\right)=\frac{1}{2}+r_{p}\left(\frac{1}{2}-\frac{2 \theta_{p}}{\pi}\right) .
$$

Example 3. Let $p_{1}=P(0.8,0.3), p_{2}=P(0.7,0.2)$ be two PFNs. According to Definitions 3-6, we have the magnitude comparison results between $p_{1}$ and $p_{2}$, as shown in Table 1 .

When we employ the score function in Eq. (5) and the closeness index in Eq. (7), we can obtain $p_{1}>p_{2}$. When we employ the scalar

Table 1 Magnitude comparison results between two PFNs.

\begin{tabular}{cccc}
\hline & $\boldsymbol{p}_{\mathbf{1}}$ & $\boldsymbol{p}_{\mathbf{2}}$ & Comparison Results \\
\hline$s(p)$ & 0.5500 & 0.4500 & $p_{1}>p_{2}$ \\
$a(p)$ & 0.8747 & 0.8670 & $p_{1}>p_{2}$ \\
$c(p)$ & 0.7165 & 0.6531 & $p_{1}<p_{2}$ \\
$V(p)$ & 0.7321 & 0.7350 & \\
\hline
\end{tabular}

PFN, Pythagorean fuzzy number. function in Eq. (7), we can obtain $p_{1}<p_{2}$. The result from the scalar function is inconsistent with the results from the score function and the closeness index. Since the scalar function takes the parameters of the direction of the commitment and the angular degree into consideration, it can reflect the unique characteristics of the PFNs, while the other three methods ignore these important parameters. Therefore, the comparison results by scalar functions are more reasonable than the results from score functions, accuracy functions, and closeness indices.

\section{NOVEL DISTANCE MEASUREMENT METHOD FOR PFNs}

In this section, we analyze several existing distance measurement methods and identify their limitations. Then, we propose a novel distance measurement method that reflects the properties of PFNs.

\subsection{Limitations of the Existing Distance Measurement Methods for PFNs}

Distance measurement is essential for fuzzy sets and has received extensive attention in the past decades. For PFNs, scholars have proposed various distance measurement methods, which are based on three main distances: the Hamming distance, the Euclidean distance, and the generalized distance.

Zhang and $\mathrm{Xu}$ [5] presented the Hamming distance measurement method for PFNs, which considers $\mu_{p}, v_{p}$, and $h_{p}$.

Definition 7. [5] Let $p_{i}=P\left(\mu_{p i}, v_{p i}\right)(i=1,2)$ be two PFNs. The normalized Hamming distance measure between $p_{1}$ and $p_{2}$ can be defined as follows:

$$
\begin{aligned}
D_{Z H}\left(p_{1}, p_{2}\right)= & \frac{1}{2}\left(\left|\left(\mu_{p 1}\right)^{2}-\left(\mu_{p 2}\right)^{2}\right|+\left|\left(v_{p 1}\right)^{2}-\left(v_{p 2}\right)^{2}\right|\right. \\
& \left.+\left|\left(h_{p 1}\right)^{2}-\left(h_{p 2}\right)^{2}\right|\right) .
\end{aligned}
$$

The parameters of Zhang and Xu's distance measurement formula are directly extended from the normalized Hamming distance measure of IF numbers [36]. Only three parameters that reflect the properties of IF numbers are considered; the unique characteristics of PFNs, such as the direction of commitment, the strength of commitment and the radian, are ignored.

Li and Zeng [32] proposed a new distance measurement equation that includes four parameters, namely, $\mu_{p}, v_{p}, r_{p}, d_{p}$, of PFNs and overcomes the main limitation of Zhang and Xu's method [5].

Definition 8. [32] Let $p_{i}=P\left(\mu_{p i}, v_{p i}\right)(i=1,2)$ be two PFNs. The normalized Hamming distance measure, the normalized Euclidean distance measure, and the normalized generalized distance measure between $p_{1}$ and $p_{2}$ can be defined, respectively, as:

$$
\begin{aligned}
D_{L H}\left(p_{1}, p_{2}\right)= & \frac{1}{4}\left(\left|\mu_{p 1}-\mu_{p 2}\right|+\left|v_{p 1}-v_{p 2}\right|\right. \\
& \left.+\left|r_{p 1}-r_{p 2}\right|+\left|d_{p 1}-d_{p 2}\right|\right), \\
D_{L E}\left(p_{1}, p_{2}\right)= & {\left[\frac { 1 } { 4 } \left(\left(\mu_{p 1}-\mu_{p 2}\right)^{2}+\left(v_{p 1}-v_{p 2}\right)^{2}\right.\right.} \\
& \left.\left.+\left(r_{p 1}-r_{p 2}\right)^{2}+\left(d_{p 1}-d_{p 2}\right)^{2}\right)\right]^{1 / 2},
\end{aligned}
$$




$$
\begin{aligned}
D_{L G}\left(p_{1}, p_{2}\right)= & {\left[\frac { 1 } { 4 } \left(\left|\mu_{p 1}-\mu_{p 2}\right|^{\lambda}+\left|\nu_{p 1}-\nu_{p 2}\right|^{\lambda}\right.\right.} \\
& \left.\left.+\left|r_{p 1}-r_{p 2}\right|^{\lambda}+\left|d_{p 1}-d_{p 2}\right|^{\lambda}\right)\right]^{1 / \lambda},
\end{aligned}
$$

where $\lambda \geq 1$.

Zeng et al. [33] incorporated the hesitation degree into the distance measurement equation that is based on Li and Zeng's method [32].

Definition 9. [33] Let $p_{i}=P\left(\mu_{p i}, v_{p i}\right)(i=1,2)$ be two PFNs. The normalized Hamming distance measure, the normalized Euclidean distance measure, and the normalized generalized distance measure between $p_{1}$ and $p_{2}$ are defined respectively as follows:

$$
\begin{aligned}
D_{Z H}^{\prime}\left(p_{1}, p_{2}\right)= & \frac{1}{5}\left(\left|\mu_{p 1}-\mu_{p 2}\right|+\left|v_{p 1}-v_{p 2}\right|+\left|h_{p 1}-h_{p 2}\right|\right. \\
& \left.+\left|r_{p 1}-r_{p 2}\right|+\left|d_{p 1}-d_{p 2}\right|\right), \\
D_{Z H}^{\prime}\left(p_{1}, p_{2}\right)= & \frac{1}{5}\left(\left(\mu_{p 1}-\mu_{p 2}\right)^{2}+\left(v_{p 1}-v_{p 2}\right)^{2}+\left(h_{p 1}-h_{p 2}\right)^{2}\right. \\
& \left.\left.+\left(r_{p 1}-r_{p 2}\right)^{2}+\left(d_{p 1}-d_{p 2}\right)^{2}\right)\right]^{1 / 2}, \\
D_{Z H}^{\prime}\left(p_{1}, p_{2}\right)= & {\left[\frac { 1 } { 5 } \left(\left|\mu_{p 1}-\mu_{p 2}\right|^{\lambda}+\left|v_{p 1}-v_{p 2}\right|^{\lambda}+\left|h_{p 1}-h_{p 2}\right|^{\lambda}\right.\right.} \\
& \left.\left.+\left|r_{p 1}-r_{p 2}\right|^{\lambda}+\left|d_{p 1}-d_{p 2}\right|^{\lambda}\right)\right]^{1 / \lambda} .
\end{aligned}
$$

where $\lambda \geq 1$.

Example 4. Let $p_{1}=P(0.8,0.3)$ and $p_{2}=P(0.7,0.2)$ be two PFNs. According to Definitions 7-9, the computed distances between $p_{1}$ and $p_{2}$ are shown as follows: $D_{Z H}\left(p_{1}, p_{2}\right)=0.2, D_{L H}\left(p_{1}, p_{2}\right)=$ $0.1043, D_{L E}\left(p_{1}, p_{2}\right)=0.1120, D_{L G}\left(p_{1}, p_{2}\right)=0.0364(\lambda=3)$, $D_{Z H}^{\prime}\left(p_{1}, p_{2}\right)=0.1087, D_{Z E}^{\prime}\left(p_{1}, p_{2}\right)=0.1150$, and $D_{Z G}^{\prime}\left(p_{1}, p_{2}\right)=$ $0.1204(\lambda=3)$. For instance,

$$
\begin{aligned}
D_{Z H}\left(p_{1}, p_{2}\right)= & 0.5 \times\left(\left|0.8^{2}-0.7^{2}\right|+\left|0.3^{2}-0.2^{2}\right|\right. \\
& \left.+\left|0.52^{2}-0.69^{2}\right|\right)=0.2,
\end{aligned}
$$

$$
\begin{aligned}
D_{L E}\left(p_{1}, p_{2}\right)= & \left(0.25 \times\left((0.8-0.7)^{2}+(0.3-0.2)^{2}\right.\right. \\
& \left.\left.+(0.85-0.73)^{2}+(0.77-0.82)^{2}\right)\right)^{1 / 2}=0.1120,
\end{aligned}
$$

and

$$
\begin{aligned}
D_{Z H}^{\prime}\left(p_{1}, p_{2}\right)= & \left(0.2 \times\left(|0.8-0.7|^{3}+|0.3-0.2|^{3}+|0.52-0.69|^{3}\right.\right. \\
& \left.\left.+|0.85-0.73|^{3}+|0.77-0.82|^{3}\right)\right)^{1 / 3}=01204 .
\end{aligned}
$$

We identify several limitations in Li and Zeng' method [32] and Zeng et al's method [33]: First, both distance measurement methods ignore the radian. According to the properties of PFNs that were analyzed by Chen [38], the angle $\theta_{p}$ in degrees plays an important role in the magnitude comparison, which determines the radians and, thus, influences the distance between two PFNs.

Second, the procedures of both distance measurement methods were simply extended from the distance measure of IF numbers [39], which employed the absolute deviations of the parameters. In line with the properties of the PFNs, the space of PFNs is larger than the space of IF numbers with the condition that $\left(\mu_{p}\right)^{2}+\left(v_{p}\right)^{2} \leq 1$. To ensure the specified scope, we should utilize the squared values of the $\mu_{p}, v_{p}, h_{p}$, and $r_{p}$ to specify their relationships. Hence, aforementioned formulas ignored the greater space of PFNs, which couldn't precisely reflect the characteristics of the PFNs.

Third, the distance measure formulas from Li and Zeng's [32] and Zeng et al. [33] didn't satisfy the basic properties of distance measure. An effective distance measurement formula of PFNs should satisfy the properties that the distance is in the range of [0,1]; however, the maximum distance values of Li and Zeng's [32] and Zeng et al.'s [33] formulas don't arrive to one. These limitations will be demonstrated in details in the subsequent comparative analysis of the distance measures for PFNs using specific cases.

\subsection{Novel Distance Measurement Method for PFNs}

To overcome the restrictions of existing distance measurement methods for PFNs, we present a novel distance measurement method that considers five parameters that represent the characteristics of PFNs and the procedure reflects the properties of PFNs.

In the novel distance measurement method, we take the parameters $\mu_{p}, v_{p}, r_{p}, d_{p}, \theta_{p}$ into the equation, but eliminate the parameter $h_{p}$.

From $\left(h_{p}\right)^{2}=1-\left(\mu_{p}\right)^{2}-\left(v_{p}\right)^{2},\left(r_{p}\right)^{2}=\left(\mu_{p}\right)^{2}+\left(v_{p}\right)^{2}$, it follows that $\left|\left(h_{p 1}\right)^{2}-\left(h_{p 2}\right)^{2}\right|=\mid\left(1-\left(\mu_{p 1}\right)^{2}-\left(\nu_{p 1}\right)^{2}\right)-\left(1-\left(\mu_{p 2}\right)^{2}-\right.$ $\left.\left(v_{p 2}\right)^{2}\right)|=|\left(\left(\mu_{p 1}\right)^{2}+\left(v_{p 1}\right)^{2}\right)-\left(\left(\mu_{p 2}\right)^{2}+\left(v_{p 2}\right)^{2}\right) \mid$, and $\left|\left(r_{p 1}\right)^{2}-\left(r_{p 2}\right)^{2}\right|=\left|\left(\left(\mu_{p 1}\right)^{2}+\left(\nu_{p 1}\right)^{2}\right)-\left(\left(\mu_{p 2}\right)^{2}+\left(\nu_{p 2}\right)^{2}\right)\right|$. Thus, $\left|\left(h_{p 1}\right)^{2}-\left(h_{p 2}\right)^{2}\right|=\left|\left(r_{p 1}\right)^{2}-\left(r_{p 2}\right)^{2}\right|$. Therefore, $\left|\left(h_{p 1}\right)^{2}-\left(h_{p 2}\right)^{2}\right|$ plays the same role as $\left|\left(r_{p 1}\right)^{2}-\left(r_{p 2}\right)^{2}\right|$ in the distance measure of PFNs. We only incorporate one of them into the distance measurement formula under the PF environment. In this paper, we select parameter $r_{p}$ in the proposed distance measurement method for PFNs.

We use $\sin \left(\theta_{p}\right)$ to represent the influence of the angle on the distance of PFNs. According to the properties of PFNs, the distance between two PFNs is influenced by the angle $\theta_{p}$. Since $\theta_{p} \in[0, \pi / 2], \sin \left(\theta_{p}\right)$ and $\cos \left(\theta_{p}\right)$ are strictly monotone, where $\sin \left(\theta_{p}\right), \cos \left(\theta_{p}\right) \in[0,1]$. They have the same properties in the distance measure.

The proposed distance measurement method for PFNs is presented below.

Definition 10. Let $p_{i}=P\left(\mu_{p i}, v_{p i}\right)(i=1,2)$ be two PFNs. The parameters of the PFNs are $\mu_{p}, v_{p}, r_{p}, d_{p}, \theta_{p}$. The normalized Hamming distance measure between $p_{1}$ and $p_{2}$ complies with the following rule: 


$$
\begin{aligned}
D\left(p_{1}, p_{2}\right)= & \frac{1}{4}\left(\left|\left(\mu_{p 1}\right)^{2}-\left(\mu_{p 2}\right)^{2}\right|+\mid\left(v_{p 1}\right)^{2}\right. \\
& -\left(v_{p 2}\right)^{2}|+|\left(r_{p 1}\right)^{2}-\left(r_{p 2}\right)^{2}|+| d_{p 1}-d_{p 2} \mid \\
& \left.+\left|\sin \left(\theta_{p 1}\right)-\sin \left(\theta_{p 2}\right)\right|\right) .
\end{aligned}
$$

Example 5. Let $p_{1}=P(0.8,0.3), p_{2}=P(0.7,0.2)$ be two PFNs. According to Definition 10, the normalized Hamming distance between $p_{1}$ and $p_{2}$ is calculated as below:

$$
\begin{aligned}
& D\left(p_{1}, p_{2}\right)=0.25 \times\left(\left|0.8^{2}-0.7^{2}\right|+\left|0.3^{2}-0.2^{2}\right|+\left|0.85^{2}-0.73^{2}\right|\right. \\
& +|0.77-0.82|+|\sin (0.36)-\sin (0.28)|)=0.1319 .
\end{aligned}
$$

Theorem 1. Let $p_{i}=P\left(\mu_{p i}, v_{p i}\right)(i=1,2)$ be two PFNs. Then, $0 \leq$ $D\left(p_{1}, p_{2}\right) \leq 1$.

Proof: It follows from $\mu_{p 1}, \mu_{p 2}, v_{p 1}, v_{p 2} \in[0,1], \theta_{p 1}, \theta_{p 2} \in$ $[0, \pi / 2], 0 \leq\left(\mu_{p 1}\right)^{2}+\left(\nu_{p 1}\right)^{2} \leq 1,0 \leq\left(\mu_{p 2}\right)^{2}+\left(v_{p 2}\right)^{2} \leq 1$, $\left(r_{p 1}\right)^{2}=\left(\mu_{p 1}\right)^{2}+\left(\nu_{p 1}\right)^{2},\left(r_{p 2}\right)^{2}=\left(\mu_{p 2}\right)^{2}+\left(\nu_{p 2}\right)^{2},\left|d_{p 1}-d_{p 2}\right|=$ $(\pi / 2) \times\left|\theta_{p 1}-\theta_{p 2}\right|,\left|d_{p 1}-d_{p 2}\right| \in[0,1]$, and $\left|\sin \left(\theta_{p 1}\right)-\sin \left(\theta_{p 2}\right)\right| \in$ $[0,1]$ that

$$
\begin{aligned}
D\left(p_{1}, p_{2}\right)= & \frac{1}{4}\left(\left|\left(\mu_{p 1}\right)^{2}-\left(\mu_{p 2}\right)^{2}\right|+\left|\left(v_{p 1}\right)^{2}-\left(v_{p 2}\right)^{2}\right|\right. \\
& +\left|\left(r_{p 1}\right)^{2}-\left(r_{p 2}\right)^{2}\right|+\left|d_{p 1}-d_{p 2}\right| \\
& \left.+\left|\sin \left(\theta_{p 1}\right)-\sin \left(\theta_{p 2}\right)\right|\right) \\
= & \frac{1}{4}\left(\left|\left(\mu_{p 1}\right)^{2}-\left(\mu_{p 2}\right)^{2}\right|+\left|\left(v_{p 1}\right)^{2}-\left(\nu_{p 2}\right)^{2}\right|\right. \\
& +\left|\left(\mu_{p 1}\right)^{2}+\left(v_{p 1}\right)^{2}\right|-\left|\left(\mu_{p 2}\right)^{2}+\left(v_{p 2}\right)^{2}\right| \\
& \left.+(2 / \pi) \times\left|\theta_{p 1}-\theta_{p 2}\right|+\left|\sin \left(\theta_{p 1}\right)-\sin \left(\theta_{p 2}\right)\right|\right) \\
\leq & \frac{1}{4}\left(\left(\mu_{p 1}\right)^{2}+\left(\nu_{p 1}\right)^{2}+\left(\mu_{p 1}\right)^{2}+\left(v_{p 1}\right)^{2}+(2 / \pi)\right. \\
& \left.\times\left|\theta_{p 1}-\theta_{p 2}\right|+\left|\sin \left(\theta_{p 1}\right)-\sin \left(\theta_{p 2}\right)\right|\right) \\
\leq & \frac{1}{4}(1+1+1+1)=1 .
\end{aligned}
$$

Moreover, all the absolute deviations in Eq. (15) are equal to or greater than zero, which completes the proof of Theorem 1.

Theorem 2. Let $p_{i}=P\left(\mu_{p i}, v_{p i}\right)(i=1,2)$ be two PFNs. Then, $D\left(p_{1}, p_{2}\right)=0$ if and only if $p_{1}=p_{2}$.

Proof: Because all the absolute deviations in Eq. (15) are equal to or greater than zero, if $D\left(p_{1}, p_{2}\right)=0$, then each absolute deviation is equal to zero.

If $\left|\left(\mu_{p 1}\right)^{2}-\left(\mu_{p 2}\right)^{2}\right|=0,\left|\left(v_{p 1}\right)^{2}-\left(v_{p 2}\right)^{2}\right|=0,\left|\left(r_{p 1}\right)^{2}-\left(r_{p 2}\right)^{2}\right|=$ $0,\left|\sin \left(\theta_{p 1}\right)-\sin \left(\theta_{p 2}\right)\right|=0,\left|d_{p 1}-d_{p 2}\right|=0, \mu_{p 1}, \mu_{p 2}, v_{p 1}, v_{p 2} \in$ $[0,1]$, and $\theta_{p 1}, \theta_{p 2} \in[0, \pi / 2]$, then $\mu_{p 1}=\mu_{p 2}, v_{p 1}=v_{p 2}, r_{p 1}=r_{p 2}$, $\theta_{p 1}=\theta_{p 2}$, and $d_{p 1}=d_{p 2}$.

Via any magnitude comparison method, we can obtain the result $p_{1}=p_{2}$, which completes the proof of Theorem 2 .

Theorem 3. Let $p_{i}=P\left(\mu_{p i}, v_{p i}\right)(i=1,2)$ be two PFNs. Then, $D\left(p_{1}, p_{2}\right)=D\left(p_{2}, p_{1}\right)$.
Proof: It follows from $\left|\left(\mu_{p 1}\right)^{2}-\left(\mu_{p 2}\right)^{2}\right|=\left|\left(\mu_{p 2}\right)^{2}-\left(\mu_{p 1}\right)^{2}\right|$, $\left|\left(v_{p 1}\right)^{2}-\left(v_{p 2}\right)^{2}\right|=\left|\left(v_{p 2}\right)^{2}-\left(v_{p 1}\right)^{2}\right|,\left|\left(r_{p 1}\right)^{2}-\left(r_{p 2}\right)^{2}\right|=$ $\left|\left(r_{p 2}\right)^{2}-\left(r_{p 1}\right)^{2}\right|,\left|d_{p 1}-d_{p 2}\right|=\left|d_{p 2}-d_{p 1}\right|$, and $\mid \sin \left(\theta_{p 1}\right)-$ $\sin \left(\theta_{p 2}\right)|=| \sin \left(\theta_{p 2}\right)-\sin \left(\theta_{p 1}\right) \mid$, that $D\left(p_{1}, p_{2}\right)=D\left(p_{2}, p_{1}\right)$, which completes the proof of Theorem 3 .

Theorem 4. Let $p_{i}=P\left(\mu_{p i}, v_{p i}\right)(i=1,2,3)$ be three PFNs. If $p_{1} \leq$ $p_{2} \leq p_{3}$, then $D\left(p_{1}, p_{2}\right) \leq D\left(p_{1}, p_{3}\right)$ and $D\left(p_{2}, p_{3}\right) \leq D\left(p_{1}, p_{3}\right)$.

Proof: According to the quasi-ordering on the space of Pythagorean membership grades from Yager [3], if $p_{1} \leq p_{2} \leq p_{3}$ we can obtain $\mu_{p 1} \leq \mu_{p 2} \leq \mu_{p 3}$ and $v_{p 1} \geq v_{p 2} \geq v_{p 3}$. Then,

$$
\begin{aligned}
\left|\left(\mu_{p 1}\right)^{2}-\left(\mu_{p 2}\right)^{2}\right| & \leq\left|\left(\mu_{p 1}\right)^{2}-\left(\mu_{p 3}\right)^{2}\right| \\
\left|\left(v_{p 1}\right)^{2}-\left(v_{p 2}\right)^{2}\right| & \leq\left|\left(v_{p 1}\right)^{2}-\left(v_{p 3}\right)^{2}\right|, \\
\left|\left(r_{p 1}\right)^{2}-\left(r_{p 2}\right)^{2}\right| & =\left|\left(\mu_{p 1}\right)^{2}+\left(v_{p 1}\right)^{2}-\left(\left(\mu_{p 2}\right)^{2}+\left(v_{p 2}\right)^{2}\right)\right| \\
& =\left|\left(\mu_{p 1}\right)^{2}-\left(\mu_{p 2}\right)^{2}+\left(v_{p 1}\right)^{2}-\left(v_{p 2}\right)^{2}\right| \\
& \leq\left|\left(\mu_{p 1}\right)^{2}-\left(\mu_{p 3}\right)^{2}+\left(v_{p 1}\right)^{2}-\left(v_{p 3}\right)^{2}\right| \\
& =\left|\left(\mu_{p 1}\right)^{2}+\left(v_{p 1}\right)^{2}-\left(\left(\mu_{p 3}\right)^{2}+\left(v_{p 3}\right)^{2}\right)\right| \\
& =\left|\left(r_{p 1}\right)^{2}-\left(r_{p 3}\right)^{2}\right| .
\end{aligned}
$$

From $\theta_{p}=\arctan \left(\nu_{p} / \mu_{p}\right), \tan \left(\theta_{p}\right)=\nu_{p} / \mu_{p}, \theta_{p} \in[0, \pi / 2]$, $\tan \left(\theta_{p 1}\right) \geq \tan \left(\theta_{p 2}\right) \geq \tan \left(\theta_{p 3}\right)$, and the function $\tan \left(\theta_{p}\right)$ being strictly monotone, it follows that $\theta_{p 1} \geq \theta_{p 2} \geq \theta_{p 3}$.

$$
\begin{aligned}
\left|d_{p 1}-d_{p 2}\right| & =(2 / \pi)\left|\theta_{p 1}-\theta_{p 2}\right| \\
& \leq(2 / \pi)\left|\theta_{p 1}-\theta_{p 3}\right| \\
& =\left|d_{p 1}-d_{p 3}\right|,
\end{aligned}
$$

and $\left|\sin \left(\theta_{p 1}\right)-\sin \left(\theta_{p 2}\right)\right| \leq\left|\sin \left(\theta_{p 1}\right)-\sin \left(\theta_{p 3}\right)\right|$. Thus,

$$
\begin{aligned}
D\left(p_{1}, p_{2}\right)= & \frac{1}{4}\left(\left|\left(\mu_{p 1}\right)^{2}-\left(\mu_{p 2}\right)^{2}\right|+\left|\left(v_{p 1}\right)^{2}-\left(v_{p 2}\right)^{2}\right|\right. \\
& +\left|\left(r_{p 1}\right)^{2}-\left(r_{p 2}\right)^{2}\right|+\left|d_{p 1}-d_{p 2}\right| \\
& \left.+\left|\sin \left(\theta_{p 1}\right)-\sin \left(\theta_{p 2}\right)\right|\right) \\
\leq & \frac{1}{4}\left(\left|\left(\mu_{p 1}\right)^{2}-\left(\mu_{p 3}\right)^{2}\right|+\left|\left(v_{p 1}\right)^{2}-\left(v_{p 3}\right)^{2}\right|\right. \\
& +\left|\left(r_{p 1}\right)^{2}-\left(r_{p 3}\right)^{2}\right|+\left|d_{p 1}-d_{p 3}\right| \\
& \left.+\left|\sin \left(\theta_{p 1}\right)-\sin \left(\theta_{p 3}\right)\right|\right) \\
= & D\left(p_{1}, p_{3}\right) .
\end{aligned}
$$

Similarly, we can also prove $D\left(p_{2}, p_{3}\right) \leq D\left(p_{1}, p_{3}\right)$, which completes the proof of Theorem 4 .

In this analysis, we have proved that the proposed normalized Hamming distance of PFNs is nonnegative, symmetric, and transitivity; moreover, the distance is in the range of $[0,1]$.

\subsection{Comparative Analysis of the Distance Measures for PFNs}

In order to illustrate the advantages of the proposed distance measure for PFNs, we conduct a comparison between the proposed distance measure and the existing distance measures from Zhang and $\mathrm{Xu}$ [5], Li and Zeng [32], and Zeng et al. [33]. We explore a series of cases expressed as PFNs based on the fundamental properties of 
the distance measure and the deviations of the magnitudes of the PFNs by scalar functions. The comparison results from different distance measures are summarized in Table 2. It is clear that the proposed distance measure can overcome the difficulties of obtaining the illogical results of the existing methods. We will investigate the following four major limitations of the existing distance measures in details.

First, the property of the distance measure in Theorem 1 is not satisfied by use of $D_{L H}, D_{L E}, D_{L G}, D_{Z H}^{\prime}, D_{Z E}^{\prime}$ and $D_{Z G}^{\prime}$ in Case 1. Because $p_{1}=P(1.00,0.00)$ and $p_{2}=P(0.00,1.00)$ are the maximum PFN and the minimum PFN by scalar functions, namely, $V(P(1.00,0.00))=1$ and $V(P(0.00,1.00))=0$, it is expected that the distance between $p_{1}=P(1.00,0.00)$ and $p_{2}=P(0.00,1.00)$ is the maximum distance, arriving to one. However, $D_{L H}=D_{L E}=$ $D_{L G}=0.75$ and $D_{Z H}^{\prime}=D_{Z E}^{\prime}=D_{Z G}^{\prime}=0.6$, none of which are up to one. The key problem is that the procedure of distance measures from Li and Zeng [32] and Zeng et al. [33] directly extended from the IF numbers, not considered the squared deviations of the parameters. Therefore, the results from Li and Zeng [32] and Zeng et al. [33] cannot ensure the greater space of the PFNs and do not satisfy the property of the distance measure in Theorem 1.

Second, the distance value $D_{Z H}=1$ in Cases 1 and 2 is unreasonable. Although they have the same $p_{1}$ in the two cases, $p_{2}=P(0.00,1.00)$ (in Case 1 ) and $p_{2}=P(1.00,0.00)$ (in Case 2 ) are obviously different. The same obtained distance values from the different PFNs are illogical and unreasonable. According to the deviations of magnitudes by the scalar function, the deviation in Case 2 is 0.5 . It is respected that the distance value is equal to or close to 0.5 , so $D_{Z H}=$ 1 is unreasonable in Case 2. The key problem is that Zhang and Xu [5] took the parameters $\mu_{p}, v_{p}$, and $h_{p}$ into the distance measure, but

Table 2 Comparison results of six cases from different distance measures for PFNs.

\begin{tabular}{cccc}
\hline & Case 1 & Case 2 & Case 3 \\
$p_{1}$ & $P(1.00,0.00)$ & $P(1.00,0.00)$ & $P(0.50,0.71)$ \\
$p_{2}$ & $P(0.00,1.00)$ & $P(0.00,0.00)$ & $P(0.00,1.00)$ \\
$V\left(p_{1}\right)$ & 1 & 1 & 0.4063 \\
$V\left(p_{2}\right)$ & 0 & 0.5 & 0 \\
\hline$D_{Z H}[5]$ & 1 & 1 & 0.5 \\
$D_{L H}[32]$ & 0.75 & 0.5 & 0.4212 \\
$D_{L E}[32]$ & 0.75 & 0.5 & 0.1849 \\
$D_{L G}[32]$ & 0.75 & 0.5 & 0.0838 \\
$D_{Z H^{\prime}}[33]$ & 0.6 & 0.6 & 0.3627 \\
$D_{Z E^{\prime}}[33]$ & 0.6 & 0.6 & 0.1515 \\
$D_{Z G^{\prime}}[33]$ & 0.6 & 0.6 & 0.0675 \\
$D($ proposed $)$ & 1 & 0.5 & 0.3938 \\
\hline & Case 4 & Case 5 & Case 6 \\
$p_{1}$ & $P(0.40,0.20)$ & $P(0.40,0.20)$ & $P(0.71,0.50)$ \\
$p_{2}$ & $P(0.50,0.30)$ & $P(0.14,0.30)$ & $P(0.00,1.00)$ \\
$V\left(p_{1}\right)$ & 0.5916 & 0.5916 & 0.5937 \\
$V\left(p_{2}\right)$ & 0.5909 & 0.4265 & 0 \\
\hline$D_{Z H}[5]$ & 0.14 & 0.1375 & 0.75 \\
$D_{L H}[32]$ & 0.0827 & 0.2018 & 0.5788 \\
$D_{L E}[32]$ & 0.0073 & 0.0606 & 0.3425 \\
$D_{L G}[32]$ & 0.0007 & 0.0214 & 0.2071 \\
$D_{Z H^{\prime}}[33]$ & 0.0934 & 0.1838 & 0.4899 \\
$D_{Z E^{\prime}}[33]$ & 0.0095 & 0.0510 & 0.2776 \\
$D_{Z G^{\prime}}[33]$ & 0.0010 & 0.0174 & 0.1662 \\
$D($ proposed $)$ & 0.0990 & 0.2830 & 0.6327 \\
\hline
\end{tabular}

PFN, Pythagorean fuzzy number.

Results in orange shaded cells represent the unreasonable distance values. ignored the $r_{p}, d_{p}$, and $\theta_{p}$ of the PFNs. This limitation will result in the same distance values from the different PFNs. The similar results occur in $D_{Z H}^{\prime}, D_{Z E}^{\prime}$, and $D_{Z G}^{\prime}$ (in Cases 1 and 2). Although Zeng et al. [33] took account of the parameters $\mu_{p}, v_{p}, h_{p}, r_{p}$, and $d_{p}$ of PFNs, they neglected $\theta_{p}$ of the PFNs. This limitation will also result in the same distance values for different PFNs. Hence, the distance measures from Zhang and $\mathrm{Xu}$ [5] and Zeng et al. [33] are demonstrated to distinguish the differences of the PFNs ineffectively in Cases 1 and 2.

Third, it is easily seen that the property of the distance measure in Theorem 4 is not satisfied by use of $D_{Z H}$ in Cases 4 and 5. Based on the magnitudes of the PFNs by scalar function, $V(P(0.40,0.20))>$ $V(P(0.40,0.20))>V(P(0.50,0.30))>V(P(0.15,0.30))$, as shown in Table 2. We obtain $(V(P(0.40,0.20))-V(P(0.50,0.30)))<$ $(V(P(0.40,0.20))-V(P(0.15,0.30)))$. It is expected that the distance value between $p_{1}=P(0.40,0.20)$ and $p_{2}=P(0.50,0.30)$ (in Case 4$)$ is greater than the distance value between $p_{1}=$ $P(0.40,0.20)$ and $p_{2}=P(0.14,0.30)$ (in Case 5). However, the $D_{Z H}$ value in Case 4 is greater than that in Case 5 , which leads to a counterintuitive result. Therefore, the distance measure from Zhang and $\mathrm{Xu}[5]$ is infeasible and unreasonable in Cases 4 and 5.

Fourth, it is clearly seen that the distance values by use of $D_{L E}, D_{L G}, D_{Z E}^{\prime}$, and $D_{Z G}^{\prime}$ in Cases 3 and 6 are unreasonable. Since $P(0.50,0.71)$ and $P(0.71,0.50)$ are compliment, they have the same $r_{p}, d_{p}$, and $\theta_{p}$. Meanwhile, in line with the magnitudes by scalar functions, $V(P(0.50,0.71))-V(P(0.00,1.00))=0.4063$ and $V(P(0.71,0.50))-V(P(0.00,1.00))=0.5937$. It is logically respected that the distance values between $p_{1}$ and $p_{2}$ are close to 0.4063 in Case 3 and 0.5937 in Case 6. However, we find out that the obtained results by use of $D_{L E}, D_{L G}, D_{Z E}^{\prime}$, and $D_{Z G}^{\prime}$ have great disparities with the expected deviations. The similar results occur in the $D_{L E}, D_{L G}, D_{Z E}^{\prime}$, and $D_{Z G}^{\prime}$ values in Cases 4 and 5 , which are obviously less than the deviations of the magnitudes. Therefore, the Euclidean distance measures and the generalized distance measures from Li and Zeng [32] and Zeng et al. [33], respectively, are not effective to measure the distance for PFNs in these cases.

Based on the comparative discussions, we find out that the distance measure from Zhang and Xu [5] couldn't precisely calculate the distance between two PFNs owing to the ignorance of some important parameters representing the characteristics of PFNs. The distance measures from Li and Zeng [31] and Zeng et al. [32] are not satisfied with the maximum value of the distance between two PFNs, because the relevant procedures do not utilize the squared deviations of corresponding parameters to ensure the greater space of the PFNs. The distance values of the Euclidean distance measure and the generalized distance measure have great disparities with the deviations of the magnitudes between the PFNs. In contrast, the proposed distance measure for PFNs can effectively conquer the difficulties of illogical results from the existing distance measures and satisfy the properties of the distance measure in Theorems 1-4. Moreover, the obtained results are closer to the deviations of the magnitudes between PFNs.

\section{PROPOSED PF-TOPSIS APPROACH FOR MCDM PROBLEMS}

In this section, we introduce the MCDM problems under the PF environment and apply the novel distance measure in the PFTOPSIS approach to deal with the MCDM problems. 


\subsection{Description of the MCDM Problems within the PF Environment}

An MCDM problem is expressed as a decision matrix, the elements of which are the assessment values of all alternatives for every criterion. Given an MCDM problem with PF information, let $X=\left\{x_{1}, x_{2}, \cdots, x_{m}\right\}(m \geq 2)$ be a set of alternatives, $C=$ $\left\{C_{1}, C_{2}, \cdots, C_{n}\right\}(n \geq 2)$ be a set of criteria, $\omega=\left(\omega_{1}, \omega_{2}, \cdots, \omega_{n}\right)^{T}$ $\left(0 \leq \omega_{j} \leq 1(j=1,2, \cdots, n)\right.$, and $\left.\sum_{j=1}^{n} \omega_{j}=1\right)$ be the weight vector for each criterion. $P\left(\mu_{p_{i j}}, v_{p_{i j}}\right)$ denotes the assessment value of the $i^{t h}$ alternative for the $j^{\text {th }}$ criterion, namely, $C_{j}\left(x_{i}\right)=$ $P\left(\mu_{p_{i j}}, v_{p_{i j}}\right)$, and $R=\left(C_{j}\left(x_{i}\right)\right)_{m \times n}$ denotes the PF decision matrix, which is concisely expressed as follows:

$$
R=\left[\begin{array}{cccc}
P\left(\mu_{p 11}, v_{p 11}\right) & P\left(\mu_{p 12}, v_{p 12}\right) & \ldots & P\left(\mu_{p 1 n}, v_{p 1 n}\right) \\
P\left(\mu_{p 21}, v_{p 21}\right) & P\left(\mu_{p 22}, v_{p 22}\right) & \ldots & P\left(\mu_{p 2 n}, v_{p 2 n}\right) \\
\vdots & \vdots & \ddots & \vdots \\
P\left(\mu_{p m 1}, v_{p m 1}\right) & P\left(\mu_{p m 2}, v_{m 2}\right) & \ldots & P\left(\mu_{p m n}, v_{p m n}\right)
\end{array}\right]
$$

\subsection{Process of the Proposed Approach}

To solve these MCDM problems within the PF environment, we propose the PF-TOPSIS approach, which is based on the principle that the optimal solution has the shortest distance from the PFPIS and the farthest distance from the PFNIS.

First, we determine the PFPIS $\left(x^{+}\right)$and the PFNIS $\left(x^{-}\right)$. The classical TOPSIS introduced the PIS and NIS, which were determined by the union operator and the intersection operator of fuzzy sets according to the properties of the criteria. The benefit criteria belong to $J_{1}$ and the cost criteria belong to $J_{2}$. Akram et al. [10] extended the PFPIS and the PFNIS from the classical TOPSIS method. Let $x_{c}^{+}$and $x_{c}^{-}$denote the classical type PFPIS and PFNIS, respectively, using the following formulas:

$$
\begin{aligned}
x_{c}^{+}= & \left\{C_{j}, \max \left(\mu_{p j}\left(x_{i}\right)\right), \min \left(\mu_{p j}\left(x_{i}\right)\right) \mid C_{j} \in J_{1}\right\}, \\
& \left\{C_{j}, \min \left(\mu_{p j}\left(x_{i}\right)\right), \max \left(\mu_{p j}\left(x_{i}\right)\right) \mid C_{j} \in J_{2}\right\} \\
= & \left\{C_{1}, P\left(\mu_{p 1}^{+}, \nu_{p 1}^{+}\right)\right\},\left\{C_{2}, P\left(\mu_{p 2}^{+}, v_{p 2}^{+}\right)\right\}, \cdots, \\
& \left\{C_{n}, P\left(\mu_{p n}^{+}, v_{p n}^{+}\right)\right\}, j=1,2, \cdots, n, \\
x_{c}^{-}= & \left\{C_{j}, \min \left(\mu_{p j}\left(x_{i}\right)\right), \max \left(\mu_{p j}\left(x_{i}\right)\right) \mid C_{j} \in J_{1}\right\}, \\
& \left\{C_{j}, \max \left(\mu_{p j}\left(x_{i}\right)\right), \min \left(\mu_{p j}\left(x_{i}\right)\right) \mid C_{j} \in J_{2}\right\} \\
= & \left\{C_{1}, P\left(\mu_{p 1}^{-}, v_{p 1}^{-}\right)\right\},\left\{C_{2}, P\left(\mu_{p 2}^{+}, v_{p 2}^{-}\right)\right\}, \cdots, \\
& \left\{C_{n}, P\left(\mu_{p n}^{-}, v_{p n}^{-}\right)\right\}, j=1,2, \cdots, n .
\end{aligned}
$$

The traditional TOPSIS also introduced the PIS and the NIS determined the fixed number 1 or 0 . Wu et al. [40] employed the PFN $P(1,0)$ or $P(0,1)$ as the fixed type PFPIS $\left(x_{f}^{+}\right)$and the fixed type PFNIS $\left(x_{f}^{-}\right)$, respectively, as follows:

$$
\begin{aligned}
& x_{f}^{+}=\left\{C_{j}, P(1,0) \mid C_{j} \in J_{1}\right\},\left\{C_{j}, P(0,1) \mid C_{j} \in J_{2}\right\}, \\
& x_{f}^{-}=\left\{C_{j}, P(0,1) \mid C_{j} \in J_{1}\right\},\left\{C_{j}, P(1,0) \mid C_{j} \in J_{2}\right\} .
\end{aligned}
$$

Zhang and $\mathrm{Xu}$ [5] developed the score function in Eq. (4) for identifying the PFPIS and the PFNIS, which narrowed the distances from the PFPIS and the PFNIS compared to the classical TOPSIS. Let $x_{s}^{+}$ and $x_{s}^{-}$denote the scored type PFPIS and PFNIS, respectively, using the following formulas:

$$
\begin{aligned}
x_{s}^{+}= & \left\{C_{j}, \max \left(s\left(C_{j}\left(x_{i}\right)\right)\right) \mid j=1,2, \cdots, n\right\} \\
= & \left\{C_{1}, P\left(\mu_{p 1}^{+}, v_{p 1}^{+}\right)\right\},\left\{C_{2}, P\left(\mu_{p 2}^{+}, v_{p 2}^{+}\right)\right\}, \cdots, \\
& \left\{C_{n}, P\left(\mu_{p n}^{+}, v_{p n}^{+}\right)\right\}, \\
x_{s}^{-}= & \left\{C_{j}, \min \left(s\left(C_{j}\left(x_{i}\right)\right)\right) \mid j=1,2, \cdots, n\right\} \\
= & \left\{C_{1}, P\left(\mu_{p 1}^{-}, v_{p 1}^{-}\right)\right\},\left\{C_{2}, P\left(\mu_{p 2}^{-}, \nu_{p 2}^{-}\right)\right\}, \cdots, \\
& \left\{C_{n}, P\left(\mu_{p n}^{-}, v_{p n}^{-}\right)\right\} .
\end{aligned}
$$

Yager [3] developed the scalar function to identify the magnitudes of the PFNs. We employ the scalar function in Eq. (7) to determine the PFPIS and the PFNIS. Let $x_{v}^{+}$and $x_{v}^{-}$denote the scalar type PFPIS and PFNIS, respectively, using the following formulas:

$$
\begin{aligned}
x_{v}^{+}= & \left\{C_{j}, \max \left(V\left(C_{j}\left(x_{i}\right)\right)\right) \mid C_{j} \in J_{1}\right\}, \\
& \left\{C_{j}, \min \left(V\left(C_{j}\left(x_{i}\right)\right)\right) \mid C_{j} \in J_{2}\right\} \\
= & \left\{C_{1}, P\left(\mu_{p 1}^{+}, v_{p 1}^{+}\right)\right\},\left\{C_{2}, P\left(\mu_{p 2}^{+}, v_{p 2}^{+}\right)\right\}, \cdots, \\
& \left\{C_{n}, P\left(\mu_{p n}^{+}, v_{p n}^{+}\right)\right\}, j=1,2, \cdots, n, \\
x_{v}^{-}= & \left\{C_{j}, \min \left(V\left(C_{j}\left(x_{i}\right)\right)\right) \mid C_{j} \in J_{1}\right\}, \\
& \left\{C_{j}, \max \left(V\left(C_{j}\left(x_{i}\right)\right)\right) \mid C_{j} \in J_{2}\right\} \\
= & \left\{C_{1}, P\left(\mu_{p 1}^{-}, v_{p 1}^{-}\right)\right\},\left\{C_{2}, P\left(\mu_{p 2}^{-}, v_{p 2}^{-}\right)\right\}, \cdots, \\
& \left\{C_{n}, P\left(\mu_{p n}^{-}, v_{p n}^{-}\right)\right\}, j=1,2, \cdots, n .
\end{aligned}
$$

Next, we calculate the distances from each alternative to the PFPIS $D\left(x_{i}, x^{+}\right)$and the PFNIS $D\left(x_{i}, x^{-}\right)$in MCDM practice because we may not find the PFPIS $\left(x^{+}\right)$or the PFNIS $\left(x^{-}\right)$if it is outside the feasible region, namely, if $x^{+}$or $x^{-} \notin X$. Moreover, in practice, decision makers typically express the importance of each element relative to the others based on various preferences. We consider $\varpi_{j}$ with respect to the importance of the criteria and propose the weighted distance measure for PFNs.

Definition 11. Let $p_{i}=\left\{P\left(\mu_{p_{i j}}, v_{p_{i j}}\right) \mid i=1,2 ; j=1,2, \cdots, n\right\}$ be two PFNs on $C=\left\{C_{1}, C_{2}, \cdots, C_{n}\right\}$. $\omega_{j}$ is the importance of criterion $j$; the vector of importances for all criteria is expressed as $\omega=\left(\omega_{1}, \omega_{2}, \cdots, \omega_{n}\right)^{T}$. The weighted distance measure equation between $p_{1}$ and $p_{2}$ is expressed as follows:

$$
\begin{aligned}
D_{W}\left(p_{1}, p_{2}\right)= & \frac{1}{4} \sum_{j=1}^{n} \omega_{j}\left(\left|\left(\mu_{p 1 j}\right)^{2}-\left(\mu_{p 2 j}\right)^{2}\right|+\mid\left(\nu_{p 1 j}\right)^{2}\right. \\
& -\left(v_{p 2 j}\right)^{2}|+|\left(r_{p 1 j}\right)^{2}-\left(r_{p 2 j}\right)^{2}|+| d_{p 1 j}-d_{p 2 j} \mid+ \\
& \left.+\left|\sin \left(\theta_{p 1 j}\right)-\sin \left(\theta_{p 2 j}\right)\right|\right),
\end{aligned}
$$

where $0 \leq \omega_{j} \leq 1(j=1,2, \ldots, n)$ and $\sum_{j=1}^{n} \omega_{j}=1$. 
Example 6. Let $p_{1}=\{P(0.8,0.3), P(0.7,0.3), P(0.6,0.3)\}$ and $p_{2}=\{P(0.7,0.2), P(0.6,0.2), P(0.8,0.2)\}$ be two sets of PFNs with respect to the set of criteria $C=\left\{C_{1}, C_{2}, C_{3}\right\}$. The weight vector of the criteria is $\omega=(0.2,0.3,0.5)^{T}$. According to Definition 11 , the weighted Hamming distance between $p_{1}$ and $p_{2}$ is calculated as follows:

$$
\begin{aligned}
& D_{W}\left(p_{1}, p_{2}\right)=0.25 \times\left(0.2 \times\left(\left|0.8^{2}-0.7^{2}\right|+\left|0.3^{2}-0.2^{2}\right|+\right.\right. \\
& \left.\left|0.85^{2}-0.73^{2}\right|+|0.77-0.82|+|\sin (0.36)-\sin (0.28)|\right)+ \\
& \left(0.3 \times\left(\left|0.7^{2}-0.6^{2}\right|+\left|0.3^{2}-0.2^{2}\right|+\left|0.76^{2}-0.63^{2}\right|+\right.\right. \\
& |0.74-0.80|+|\sin (0.40)-\sin (0.32)|)+\left(0.5 \times\left(\mid 0.6^{2}-\right.\right. \\
& 0.8^{2}|+| 0.3^{2}-0.2^{2}|+| 0.67^{2}-0.82^{2}|+| 0.70-0.84 \mid \\
& +|\sin (0.46)-\sin (0.24)|))=0.2439
\end{aligned}
$$

Theorem 5. Let $p_{i}=\left\{P\left(\mu_{p_{i j}}, v_{p_{i j}}\right) \mid i=1,2 ; j=1,2, \cdots, n\right\}$ be two PFNs on $C=\left\{C_{1}, C_{2}, \cdots, C_{n}\right\}$. The vector of importance for all criteria is expressed as $\omega=\left(\omega_{1}, \omega_{2}, \cdots, \omega_{n}\right)^{T}$. Then, $0 \leq D_{W}\left(p_{1}, p_{2}\right) \leq 1$.

Proof: Since $0 \leq \omega_{j} \leq 1(j=1,2, \ldots, n)$ and $\sum_{j=1}^{n} \omega_{j}=1$, if $D_{W H}\left(p_{1}, p_{2}\right)=1$, we should set the distance of each criterion between two PFNs to $D\left(p_{1}, p_{2}\right)=1$; if $D_{W H}\left(p_{1}, p_{2}\right)=0$, we should also set it to $D\left(p_{1}, p_{2}\right)=0$. Since all the normalized Hamming distances of PFNs satisfy the properties that $D\left(p_{1}, p_{2}\right) \in$ $[0,1]$, we obtain $D_{W}\left(p_{1}, p_{2}\right) \in[0,1]$, which completes the proof of Theorem 5 .

Theorem 6. Let $p_{i}=\left\{P\left(\mu_{p_{i j}}, v_{p_{i j}}\right) \mid i=1,2 ; j=1,2, \cdots, n\right\}$ be two PFNs on $C=\left\{C_{1}, C_{2}, \cdots, C_{n}\right\}$. The vector of importance for all criteria is expressed as $\omega=\left(\omega_{1}, \omega_{2}, \cdots, \omega_{n}\right)^{T}$. Then, $D_{W}\left(p_{1}, p_{2}\right)=0$ if and only if $p_{1}=p_{2}$.

Proof: Since all the absolute deviations in Eq. (25) are equal to or greater than zero, if $D_{W}\left(p_{1}, p_{2}\right)=0$, each absolute deviation is equal to zero. In addition, $\mu_{p 1 j}, \mu_{p 2 j}, v_{p 1 j}, v_{p 2 j}, r_{p 1 j}, r_{p 2 j} \in[0,1]$, $\theta_{p 1 j}$, and $\theta_{p 2 j} \in[0, \pi / 2]$. Therefore, $\mu_{p 1 j}=\mu_{p 2 j}, v_{p 1 j}=v_{p 2 j}$, $r_{p 1 j}=r_{p 2 j}, \theta_{p 1 j}=\theta_{p 2 j}$, and $d_{p 1 j}=d_{p 2 j}$. Employing any magnitude comparison method, we can obtain $p_{1}=p_{2}$ under the same criterion, which completes the proof of Theorem 6 .

Theorem 7. Let $p_{i}=\left\{P\left(\mu_{p_{i j}}, v_{p_{i j}}\right) \mid i=1,2 ; j=1,2, \cdots, n\right\}$ be two PFNs on $C=\left\{C_{1}, C_{2}, \cdots, C_{n}\right\}$. The vector of importance for all criteria is expressed as $\omega=\left(\omega_{1}, \omega_{2}, \cdots, \omega_{n}\right)^{T}$. Then, $D_{W}\left(p_{1}, p_{2}\right)=$ $D_{W}\left(p_{2}, p_{1}\right)$.

Theorem 8. Let $p_{i}=\left\{P\left(\mu_{p_{i j}}, v_{p_{i j}}\right) \mid i=1,2,3 ; j=1,2, \cdots, n\right\}$ be three PFNs on $C=\left\{C_{1}, C_{2}, \cdots, C_{n}\right\}$. The vector of importance for all criteria is expressed as $\omega=\left(\omega_{1}, \omega_{2}, \cdots, \omega_{n}\right)^{T}$. Then, $D_{W}\left(p_{1}, p_{2}\right) \leq$ $D_{W}\left(p_{1}, p_{3}\right)$ and $D_{W}\left(p_{2}, p_{3}\right) \leq D_{W}\left(p_{1}, p_{3}\right)$.

Proof: In line with Theorem 4 , if $p_{1} \leq p_{2} \leq p_{3}$ for each criterion, we can obtain $\mu_{p 1} \leq \mu_{p 2} \leq \mu_{p 3}$ and $\nu_{p 1} \geq v_{p 2} \geq v_{p 3}$. Then, $D\left(p_{1}, p_{2}\right) \leq D\left(p_{1}, p_{3}\right)$ and $D\left(p_{2}, p_{3}\right) \leq D\left(p_{1}, p_{3}\right)$. For two PFNs on $C=\left\{C_{1}, C_{2}, \cdots, C_{n}\right\}, D_{W}\left(p_{1}, p_{2}\right)$ is equal to the weighted sum of each $D\left(p_{1}, p_{2}\right)$. Since the weight of the $j^{t h}$ criterion is the same, $D_{W}\left(p_{1}, p_{2}\right) \leq D_{W}\left(p_{1}, p_{3}\right)$ remains satisfied.

Similarly, we can also prove $D_{W}\left(p_{2}, p_{3}\right) \leq D_{W}\left(p_{1}, p_{3}\right)$ by the same way, which completes the proof of Theorem 8 .
We obtain the distance of alternative $x_{i}$ from PFPIS $\left(x^{+}\right)$based on Eq. (25), which can be defined as follows:

$$
\begin{aligned}
D\left(x_{i}, x^{+}\right)= & \sum_{j=1}^{n} \omega_{j} D\left(C_{j}\left(x_{i}\right), C_{j}\left(x^{+}\right)\right) \\
= & \frac{1}{4} \sum_{j=1}^{n} \omega_{j}\left(\left|\left(\mu_{p i j}\right)^{2}-\left(\mu_{p j}^{+}\right)^{2}\right|+\left|\left(v_{p i j}\right)^{2}-\left(v_{p j}^{+}\right)^{2}\right|\right. \\
& +\left|\left(r_{p i j}\right)^{2}-\left(r_{p j}^{+}\right)^{2}\right|+\left|d_{p i j}-d_{p j}^{+}\right|+\mid \sin \left(\theta_{p i j}\right) \\
& \left.-\sin \left(\theta_{p j}^{+}\right) \mid\right),
\end{aligned}
$$

where $i=1,2, \cdots, m$ and $x^{+}$represents $x_{c}^{+}, x_{f}^{+}$or $x_{v}^{+}$.

According to the principle of TOPSIS, the smaller $D\left(x_{i}, x^{+}\right)$is, the better the alternative $x_{i}$ is. Let:

$$
D_{\min }\left(x_{i}, x^{+}\right)=\min _{i=1}^{m} D\left(x_{i}, x^{+}\right) .
$$

The distance between alternative $x_{i}$ and the PFNIS $\left(x^{-}\right)$can be defined as follows:

$$
\begin{aligned}
D\left(x_{i}, x^{-}\right)= & \sum_{j=1}^{n} \omega_{j} D\left(C_{j}\left(x_{i}\right), C_{j}\left(x^{-}\right)\right) \\
= & \frac{1}{4} \sum_{j=1}^{n} \omega_{j}\left(\left|\left(\mu_{p i j}\right)^{2}-\left(\mu_{p j}^{-}\right)^{2}\right|+\left|\left(v_{p i j}\right)^{2}-\left(\nu_{p j}^{-}\right)^{2}\right|\right. \\
& +\left|\left(r_{p i j}\right)^{2}-\left(r_{p j}^{-}\right)^{2}\right|+\left|d_{p i j}-d_{p j}^{-}\right|+\mid \sin \left(\theta_{p i j}\right) \\
& \left.-\sin \left(\theta_{p j}^{-}\right) \mid\right),
\end{aligned}
$$

where $i=1,2, \cdots, m$ and $x^{-}$represents $x_{c}^{-}, x_{f}^{-}$or $x_{v}^{-}$.

According to the principle of TOPSIS, the larger $D\left(x_{i}, x^{-}\right)$is, the better the alternative $x_{i}$ is. Let:

$$
D_{\max }\left(x_{i}, x^{-}\right)=\max _{i=1}^{m} D\left(x_{i}, x^{-}\right) .
$$

Traditionally, we calculate the $R C$ of the alternative $x_{i}$ with respect to the PFPIS $\left(x^{+}\right)$and the PFNIS $\left(x^{-}\right)$in line with the basic principle of classical TOPSIS. The formula for $R C\left(x_{i}\right)$ is expressed as follows:

$$
R C\left(x_{i}\right)=\frac{D\left(x_{i}, x^{-}\right)}{D\left(x_{i}, x^{+}\right)+D\left(x_{i}, x^{-}\right)} .
$$

In view of Hadi-Vencheh and Mirjaberi's formula [41], according to which the optimal solution has the shortest distance from the PIS and the farthest distance from the NIS, concurrently, Zhang and $\mathrm{Xu}$ [5] and Akram et al. [10] utilized a revised index, which is denoted as $\zeta\left(x_{i}\right)$, to identify the ranking order, which is expressed as follows:

$$
\zeta\left(x_{i}\right)=\frac{D\left(x_{i}, x^{-}\right)}{D_{\max }\left(x_{i}, x^{-}\right)}-\frac{D\left(x_{i}, x^{+}\right)}{D_{\min }\left(x_{i}, x^{+}\right)} .
$$

$R C\left(x_{i}\right)$ is the classical index for determining the ranking order of alternatives, which has been extensively used and can reflect the performance of TOPSIS. The index $\zeta\left(x_{i}\right)$ considers the optimal solution to be close to PFPIS and far from PFNIS simultaneously. To examine the feasibility of the proposed approach, we adopt both indices in this paper. According to $R C\left(x_{i}\right)$ or $\zeta\left(x_{i}\right)$, we obtain the 
ranking order of alternatives $x_{i}$, which we use to determine the optimal solution according to the maximum value of $R C\left(x_{i}\right)$ or $\zeta\left(x_{i}\right)$.

$$
\begin{gathered}
x^{*}=\left\{x_{i}:\left(i=R C\left(x_{i}\right)=\max _{i=1}^{m} R C\left(x_{i}\right)\right)\right\}, \\
x^{* \prime}=\left\{x_{i}:\left(i=\zeta\left(x_{i}\right)=\max _{i=1}^{m} \zeta\left(x_{i}\right)\right)\right\} .
\end{gathered}
$$

\subsection{Algorithm of the Proposed Approach}

In line with the above analysis, the proposed PF-TOPSIS approach is separated into four paths for identifying the PFPIS and the PFNIS: the classical type applies the classical TOPSIS union operator or intersection operator; the fixed type applies the fixed PFNs of $P(1,0)$ or $P(0,1)$; the scored type applies the score function; the scalar type applies the scalar function. We describe the algorithm of the proposed PF-TOPSIS method in the following seven steps. We present a flowchart that illustrates the process of the proposed approach in Fig. 2.

Step 1. Construct a PF decision matrix $R=\left(C_{j}\left(x_{i}\right)\right)_{m \times n}$ for an MCDM problem under the PF environment, where each element $C_{j}\left(x_{i}\right)$ is the assessment value of the $i^{\text {th }}$ alternative with respect to the $j^{\text {th }}$ criterion.

Step 2. Identify the PFPIS. Utilize Eqs. (17), (19), (21), and (23) to identify the PFPIS $x^{+}=\left\{C_{1}\left(x^{+}\right), C_{2}\left(x^{+}\right), \cdots, C_{\mathrm{n}}\left(x^{+}\right)\right\}\left(x^{+}\right.$represents $x_{c}^{+}, x_{f}^{+}, x_{s}^{+}$or $\left.x_{v}^{+}\right)$for the classical type, the fixed type, the scored type, and the scalar type, respectively.

Step 3. Identify the PFNIS. Utilize Eqs. (18), (20), (22), and (24) to identify the PFNIS $x^{-}=\left\{C_{1}\left(x^{-}\right), C_{2}\left(x^{-}\right), \cdots, C_{\mathrm{n}}\left(x^{-}\right)\right\}\left(x^{-}\right.$represents $x_{c}^{-}, x_{f}^{-}, x_{s}^{-}$or $\left.x_{v}^{-}\right)$for the classical type, the fixed type, the scored type, and the scalar type, respectively.

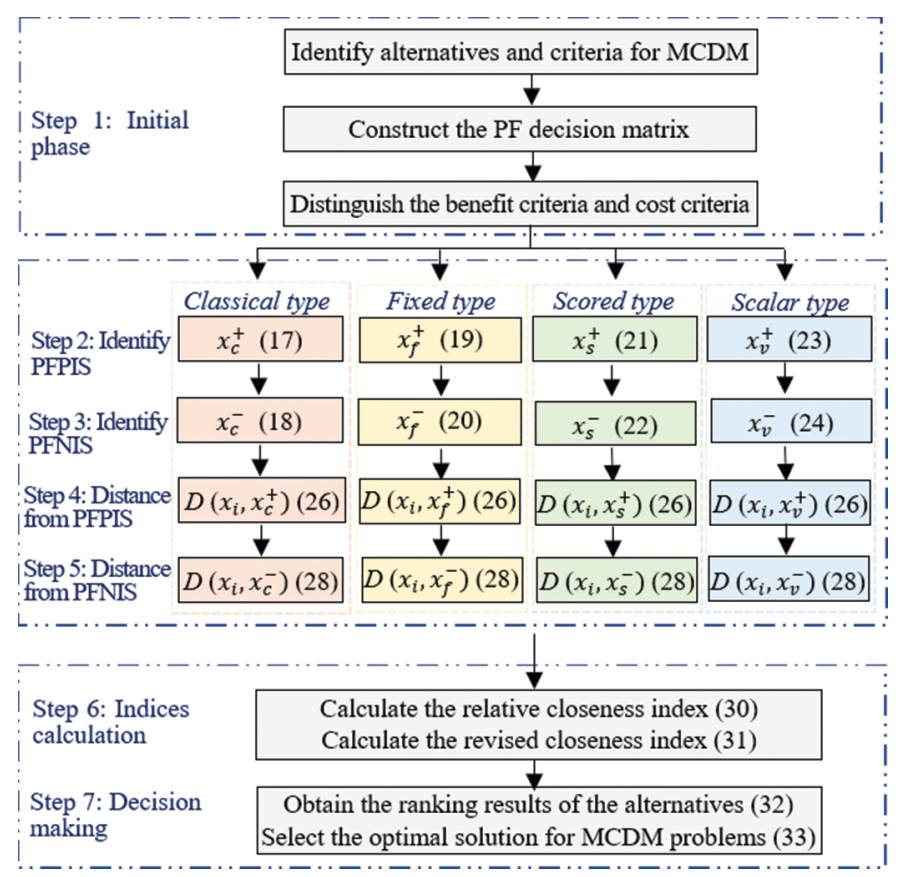

Figure 2 Flowchart of the proposed Pythagorean fuzzy technique for order preference by similarity to ideal solutions (PF-TOPSIS) approach for multicriteria decision-making (MCDM) problems.
Step 4. Employ Eq. (26) to calculate the distance of alternative $x_{i}$ from the PFPIS and use Eq. (27) to determine the minimum distance from the PFPIS.

Step 5. Employ Eq. (28) to calculate the distance of alternative $x_{i}$ from the PFNIS and use Eq. (29) to determine the maximum distance from the PFNIS.

Step 6. Utilize Eqs. (30) and (31) to compute the RC index $R C\left(x_{i}\right)$ and the revised closeness index $\zeta\left(x_{i}\right)$, respectively, of alternative $x_{i}$.

Step 7. Employ Eqs. (32) and (33) to obtain the ranking order of alternatives $x_{i}$ and identify the optimal solution, which corresponds to the maximum value of $R C\left(x_{i}\right)$ or $\zeta\left(x_{i}\right)$.

\section{ILLUSTRATIVE EXAMPLE}

In this section, we consider an example evaluation for emerging technology commercialization that was adapted from Wei and $\mathrm{Lu}$ [42] as an MCDM problem on which to evaluate the feasibility of the proposed approaches and conduct a comparison analysis among four types of approaches under the PF environment.

\subsection{Description of the Example}

The example from Wei and $\mathrm{Lu}$ [42] was used to evaluate the commercialization of emerging technology companies. Five potential emerging technology companies are expressed as a set of alternatives $X=\left\{x_{1}, x_{2}, x_{3}, x_{4}, x_{5}\right\}$. The experts selected four major criteria $\left(C_{1}\right.$ : the technical advancement; $C_{2}$ : the potential market risk; $C_{3}$ : the industrialization infrastructure, human resources, and financial conditions; and $C_{4}$ : the employment criterion and the development of science and technology), which are expressed as a set of criteria $C=\left\{C_{1}, C_{2}, C_{3}, C_{4}\right\}$. All the criteria are benefit attributes. The weight vector of the criteria that is provided by the decision makers is expressed as $\omega=(0.2,0.1,0.3,0.4)^{T}$.

The assessment values of the five companies with respect to the four criteria that were specified by the decision makers are expressed as PFNs, as listed in Table 3. For example, the element $C_{1}\left(x_{1}\right)=$ $P(0.5,0.8)$ specifies that the degree to which alternative $x_{1}$ satisfies criterion $C_{1}$ is 0.5 and the degree to which alternative $x_{1}$ dissatisfies criterion $C_{1}$ is 0.8 .

\subsection{Decision Process of the Proposed Approach}

According to the algorithm of the proposed PF-TOPSIS approach, we apply the proposed PF-TOPSIS approach to deal with the MCDM problem that is discussed in Subsection 5.1.

Table 3 PF decision matrix that was specified by the decision maker.

\begin{tabular}{ccccc}
\hline & $\boldsymbol{C}_{\mathbf{1}}$ & $\boldsymbol{C}_{\mathbf{2}}$ & $\boldsymbol{C}_{\mathbf{3}}$ & $\boldsymbol{C}_{\mathbf{4}}$ \\
\hline$x_{\mathbf{1}}$ & $P(0.5,0.8)$ & $P(0.6,0.3)$ & $P(0.3,0.6)$ & $P(0.5,0.7)$ \\
$x_{\mathbf{2}}$ & $P(0.7,0.5)$ & $P(0.7,0.2)$ & $P(0.9,0.2)$ & $P(0.8,0.5)$ \\
$x_{3}$ & $P(0.6,0.2)$ & $P(0.5,0.2)$ & $P(0.5,0.3)$ & $P(0.6,0.3)$ \\
$x_{\mathbf{4}}$ & $P(0.4,0.2)$ & $P(0.6,0.3)$ & $P(0.3,0.4)$ & $P(0.5,0.4)$ \\
$x_{5}$ & $P(0.6,0.4)$ & $P(0.4,0.8)$ & $P(0.7,0.6)$ & $P(0.5,0.8)$ \\
\hline
\end{tabular}

PF, Pythagorean fuzzy. 
Step 1. We utilize Eqs. (17) and (18) to calculate the classical type PFPIS $\left(x_{c}^{+}\right)$and the classical type PFNIS $\left(x_{c}^{-}\right)$, respectively. Then we obtain the results of $x_{c}^{+}$and $x_{c}^{-}$as follows:

$$
\begin{aligned}
& x_{c}^{+}=\{P(0.7,0.2), P(0.7,0.2), P(0.9,0.2), P(0.8,0.3)\}, \\
& x_{c}^{-}=\{P(0.4,0.8), P(0.4,0.8), P(0.3,0.6), P(0.5,0.8)\} .
\end{aligned}
$$

We utilize Eqs. (19) and (20) to calculate the fixed type PFPIS $\left(x_{f}^{+}\right)$ and the fixed type PFNIS $\left(x_{f}^{-}\right)$, respectively. We obtain the results of $x_{f}^{+}$and $x_{f}^{-}$as follows:

$$
\begin{aligned}
& x_{f}^{+}=\{P(1.0,0.0), P(1.0,0.0), P(1.0,0.0), P(1.0,0.0)\}, \\
& x_{f}^{-}=\{P(0.0,1.0), P(0.0,1.0), P(0.0,1.0), P(0.0,1.0)\} .
\end{aligned}
$$

We utilize Eqs. (21) and (22) to calculate the scored type PFPIS $\left(x_{s}^{+}\right)$ and the scored type PFNIS $\left(x_{s}^{-}\right)$, respectively. We obtain the results of $x_{s}^{+}$and $x_{s}^{-}$as follows:

$$
\begin{aligned}
& x_{s}^{+}=\{P(0.6,0.2), P(0.6,0.3), P(0.9,0.2), P(0.8,0.5)\}, \\
& x_{s}^{-}=\{P(0.5,0.8), P(0.4,0.8), P(0.3,0.6), P(0.5,0.8)\} .
\end{aligned}
$$

We utilize Eqs. (23) and (24) to calculate the scalar type PFPIS $\left(x_{v}^{+}\right)$ and the scalar type PFNIS $\left(x_{v}^{-}\right)$, respectively. Then, we obtain the results of $x_{v}^{+}$and $x_{v}^{-}$as follows:

$$
\begin{aligned}
& x_{v}^{+}=\{P(0.6,0.2), P(0.5,0.2), P(0.9,0.2), P(0.6,0.3)\}, \\
& x_{v}^{-}=\{P(0.5,0.8), P(0.4,0.8), P(0.3,0.6), P(0.5,0.8)\} .
\end{aligned}
$$

Step 2. We utilize Eqs. (26) and (27) and Eqs. (28) and (29) to calculate the distances of each alternative $x_{i}$ from the PFPIS as well as the PFNIS, respectively. The results of $D\left(x_{i}, x_{c}^{+}\right)$and $D\left(x_{i}, x_{c}^{-}\right), D\left(x_{i}, x_{f}^{+}\right)$and $D\left(x_{i}, x_{f}^{-}\right), D\left(x_{i}, x_{s}^{+}\right)$and $D\left(x_{i}, x_{s}^{-}\right)$, as well as $D\left(x_{i}, x_{v}^{+}\right)$and $D\left(x_{i}, x_{v}^{-}\right)$are listed in Tables $4-7$, respectively. Alternative $x_{2}$ has the shortest distance from PFPIS for the classical type, the fixed type, and the scored type approaches, whereas $x_{3}$ does for the scalar type approach. Meanwhile, alternative $x_{3}$ has the farthest distance from the PFNIS for all types of approaches.

Step 3. We employ Eqs. (30) and (31) to compute the $R C\left(x_{i}\right)$ and the $\zeta\left(x_{i}\right)$ for each alternative $x_{i}$ from four types of approaches, which are also listed in Tables 4-7.

Step 4. We obtain the ranking order of the five alternatives in accordance of the results from $R C\left(x_{i}\right)$ and $\zeta\left(x_{i}\right)$, which are listed in Tables $4-7$.

The ranking results that are based on $R C\left(x_{i}\right)$ and $\zeta\left(x_{i}\right)$ in this example are completely consistent from classical type, fixed type, and scored type approaches, namely, $x_{2}>x_{3}>x_{4}>x_{5}>x_{1}$, among

Table 4 Results that were obtained from the classical type PF-TOPSIS approach.

\begin{tabular}{ccccc}
\hline & $\boldsymbol{D}\left(\boldsymbol{x}_{\boldsymbol{i}}, \boldsymbol{x}_{\boldsymbol{c}}^{+}\right)$ & $\boldsymbol{D}\left(\boldsymbol{x}_{\boldsymbol{i}}, \boldsymbol{x}_{\boldsymbol{c}}^{-}\right)$ & $\boldsymbol{R C}\left(\boldsymbol{x}_{\boldsymbol{i}}\right)($ Rank) & $\boldsymbol{\zeta}\left(\boldsymbol{x}_{\boldsymbol{i}}\right)$ (Rank) \\
\hline$x_{1}$ & 0.5116 & 0.1740 & $0.2538(5)$ & $-2.6807(5)$ \\
$x_{2}$ & 0.1688 & 0.4978 & $0.7467(1)$ & $0.0000(1)$ \\
$x_{3}$ & 0.2505 & 0.5316 & $0.6797(2)$ & $-0.4160(2)$ \\
$x_{4}$ & 0.3930 & 0.4106 & $0.5110(3)$ & $-1.5026(3)$ \\
$x_{5}$ & 0.4888 & 0.2827 & $0.3665(4)$ & $-2.3271(4)$ \\
\hline
\end{tabular}

PF-TOPSIS, Pythagorean fuzzy technique for order preference by similarity to ideal solutions; RC, relative closeness.
Table 5 Results that were obtained from the fixed type PF-TOPSIS approach.

\begin{tabular}{lcclc}
\hline & $\boldsymbol{D}\left(\boldsymbol{x}_{\boldsymbol{i}}, \boldsymbol{x}_{\boldsymbol{f}}^{+}\right)$ & $\boldsymbol{D}\left(\boldsymbol{x}_{\boldsymbol{i}}, \boldsymbol{x}_{\boldsymbol{f}}^{-}\right)$ & $\boldsymbol{R} \boldsymbol{C}\left(\boldsymbol{x}_{\boldsymbol{i}}\right)($ Rank $)$ & $\boldsymbol{\zeta}\left(\boldsymbol{x}_{\boldsymbol{i}}\right)($ Rank $)$ \\
\hline$x_{1}$ & 0.8243 & 0.6900 & $0.4556(5)$ & $-1.0036(5)$ \\
$x_{2}$ & 0.4403 & 0.7945 & $0.6434(1)$ & $0.0000(1)$ \\
$x_{3}$ & 0.6013 & 0.8636 & $0.5895(2)$ & $-0.2780(2)$ \\
$x_{4}$ & 0.7437 & 0.8436 & $0.5315(3)$ & $-0.6270(3)$ \\
$x_{5}$ & 0.7738 & 0.6797 & $0.4676(4)$ & $-0.9017(4)$ \\
\hline
\end{tabular}

PF-TOPSIS, Pythagorean fuzzy technique for order preference by similarity to ideal solutions; RC, relative closeness.

Table 6 Results that were obtained from the scored type PF-TOPSIS approach.

\begin{tabular}{ccccc}
\hline & $\boldsymbol{D}\left(\boldsymbol{x}_{\boldsymbol{i}}, \boldsymbol{x}_{\boldsymbol{s}}^{+}\right)$ & $\boldsymbol{D}\left(\boldsymbol{x}_{\boldsymbol{i}}, \boldsymbol{x}_{\boldsymbol{s}}^{-}\right)$ & $\boldsymbol{R C}\left(\boldsymbol{x}_{\boldsymbol{i}}\right)($ Rank $)$ & $\zeta\left(\boldsymbol{x}_{\boldsymbol{i}}\right)$ (Rank) \\
\hline$x_{1}$ & 0.4411 & 0.1597 & $0.2657(5)$ & $-3.7959(5)$ \\
$x_{2}$ & 0.1076 & 0.4924 & $0.8207(1)$ & $-0.0642(1)$ \\
$x_{3}$ & 0.2521 & 0.5262 & $0.6761(2)$ & $-1.3423(2)$ \\
$x_{4}$ & 0.3295 & 0.4123 & $0.5570(3)$ & $-2.2747(3)$ \\
$x_{5}$ & 0.3973 & 0.2215 & $0.3579(4)$ & $-3.2713(4)$ \\
\hline
\end{tabular}

PF-TOPSIS, Pythagorean fuzzy technique for order preference by similarity to ideal solutions; RC, relative closeness.

Table 7 Results that were obtained from the scalar type PF-TOPSIS approach.

\begin{tabular}{ccccc}
\hline & $\boldsymbol{D}\left(\boldsymbol{x}_{\boldsymbol{i}}, \boldsymbol{x}_{\boldsymbol{v}}^{+}\right)$ & $\boldsymbol{D}\left(\boldsymbol{x}_{\boldsymbol{i}}, \boldsymbol{x}_{\boldsymbol{v}}^{-}\right)$ & $\boldsymbol{R C}\left(\boldsymbol{x}_{\boldsymbol{i}}\right)$ (Rank) & $\boldsymbol{\zeta}\left(\boldsymbol{x}_{\boldsymbol{i}}\right)$ (Rank) \\
\hline$x_{1}$ & 0.4855 & 0.1597 & $0.2475(5)$ & $-1.7367(5)$ \\
$x_{2}$ & 0.2380 & 0.4924 & $0.6742(2)$ & $-0.0642(2)$ \\
$x_{3}$ & 0.1217 & 0.5262 & $0.8122(1)$ & $0.4887(1)$ \\
$x_{4}$ & 0.2979 & 0.4143 & $0.5817(3)$ & $-0.4644(3)$ \\
$x_{5}$ & 0.4579 & 0.2774 & $0.3772(4)$ & $-1.3971(4)$ \\
\hline
\end{tabular}

PF-TOPSIS, Pythagorean fuzzy technique for order preference by similarity to ideal solutions; RC, relative closeness.

which $x_{2}$ is the best alternative. However, the ranking results from the scalar type approach is $x_{3}>x_{2}>x_{4}>x_{5}>x_{1}$, among which $x_{3}$ is the best alternative.

\subsection{Comparative Analysis}

The main differences among the approaches are the identification of the PFPIS, the PFNIS, and the distance measure of the alternative from the PFPIS and the PFNIS according to the previously described analysis. Therefore, we compare the scalar type PF-TOPSIS with the classical type PF-TOPSIS and the fixed type PF-TOPSIS to analyze how the PFPIS and the PFNIS influence the ranking order results for the same distance measure. Meanwhile, we compare the scalar type approach with the scored type approach that employs the distance measure from Zhang and Xu [5] to analyze the influence of the PFPIS, the PFNIS, and the distance measure on the ranking order results.

First, we calculate the PFPIS and the PFNIS via the above four approaches. The PFPIS $\left(x^{+}\right)$and PFNIS $\left(x^{-}\right)$results are listed in Table 8.

The differences between the results of $x^{+}$and $x^{-}$that were obtained via the methods of the scored type and the scalar type are smaller compared to the methods of the classical type and the fixed type, as listed in Table 8. Therefore, the results of the scored type and the 
scalar type narrow the range of the ideal solution, which directly influence the distance from $x^{+}$and $x^{-}$. In addition, the main difference between the methods of the scored type and the scalar type depends mainly on $C_{2}$ and $C_{4}$ with respect to $x^{+}$; we should further compare the magnitudes of three PFNs: $P(0.5,0.2), P(0.6,0.3)$, and $P(0.8,0.5)$.

According to Li and Zeng [32] and Zeng et al. [33], the magnitude comparison methods from score functions are extended directly from IF numbers. Considering the properties of PFNs, especially the direction of commitment and the angle in degrees, they compared the effectiveness of the magnitude measure between the score function and the scalar function that was based on the same PFN. They concluded that the scalar function was more accurate. Chen [39] further analyzed the desirable and important properties of scalar function $V(p): V(p) \in[0,1] ; V(p)=0.5$ if $\left(r_{p}, \theta_{p}\right)=$ $(0, \pi / 4) ; V(p)=1$ if $\left(r_{p}, \theta_{p}\right)=(1,0) ; V(p)$ decreases as $\theta_{p}$ increases if $r_{p}$ is fixed; $V(p)$ increases as $r_{p}$ increases if $\theta_{p}$ is fixed and $\theta_{p}<\pi / 4 ; V(p)$ decreases as $r_{p}$ increases if $\theta_{p}$ is fixed and $\theta_{p}>$ $\pi / 4$. Considering the unique properties of the PFNs, the scalar function is more suitable for magnitude comparison of PFNs than the score function. We obtain $V(P(0.5,0.2)=0.6388, V(P(0.6,0.3)=$ 0.6374 , and $V(P(0.8,0.5)=0.6362$; the magnitude comparison results, namely, $P(0.5,0.2)>P(0.6,0.3)>P(0.8,0.5)$, are reasonable, which precisely reflect the PFPIS. Additionally, the results of $x^{-}$that are obtained via the scored type approach are the same as those of the scalar type approach.

Second, we calculate the distances $D\left(x_{i}, x^{+}\right)$between alternative $x_{i}$ and the PFPIS and the distances $D\left(x_{i}, x^{-}\right)$between alternative $x_{i}$ and the PFNIS, which are reported in Tables 9 and 10, respectively.

To facilitate understanding, we present the $D\left(x_{i}, x^{+}\right)$and $D\left(x_{i}, x^{-}\right)$ comparison results graphically in Figs. 3 and 4, respectively. The

Table 8 PFPIS and PFNIS results that are based on four PF-TOPSIS approaches.

\begin{tabular}{ccccc}
\hline $\boldsymbol{x}^{+}$ & $\boldsymbol{C}_{\mathbf{1}}$ & $\boldsymbol{C}_{\mathbf{2}}$ & $\boldsymbol{C}_{\mathbf{3}}$ & $\boldsymbol{C}_{\mathbf{4}}$ \\
\hline I & $P(0.7,0.2)$ & $P(0.7,0.2)$ & $P(0.9,0.2)$ & $P(0.8,0.3)$ \\
II & $P(1.0,0.0)$ & $P(1.0,0.0)$ & $P(1.0,0.0)$ & $P(1.0,0.0)$ \\
III & $P(0.6,0.2)$ & $P(0.6,0.3)$ & $P(0.9,0.2)$ & $P(0.8,0.5)$ \\
IV & $P(0.6,0.2)$ & $P(0.5,0.2)$ & $P(0.9,0.2)$ & $P(0.6,0.3)$ \\
\hline $\boldsymbol{x}^{-}$ & $\boldsymbol{C}_{\mathbf{1}}$ & $\boldsymbol{C}_{\mathbf{2}}$ & $\boldsymbol{C}_{\mathbf{3}}$ & $\boldsymbol{C}_{\boldsymbol{4}}$ \\
\hline I & $P(0.4,0.8)$ & $P(0.4,0.8)$ & $P(0.3,0.6)$ & $P(0.5,0.8)$ \\
II & $P(0.0,1.0)$ & $P(0.0,1.0)$ & $P(0.0,1.0)$ & $P(0.0,1.0)$ \\
III & $P(0.5,0.8)$ & $P(0.4,0.8)$ & $P(0.3,0.6)$ & $P(0.5,0.8)$ \\
IV & $P(0.5,0.8)$ & $P(0.4,0.8)$ & $P(0.3,0.6)$ & $P(0.5,0.8)$ \\
\hline
\end{tabular}

PFNIS, Pythagorean fuzzy negative ideal solution; PFPIS, Pythagorean fuzzy positive ideal solution; PF-TOPSIS, Pythagorean fuzzy technique for order preference by similarity to ideal solutions.

I: The classical type PF-TOPSIS;

II: The fixed type PF-TOPSIS;

III: The scored type PF-TOPSIS;

IV: The scalar type PF-TOPSIS.

Table $9 \mid D\left(x_{i}, x^{+}\right)$results based on four approaches.

\begin{tabular}{llccc}
\hline & I (Rank) & II (Rank) & III (Rank) & IV (Rank) \\
\hline$x_{1}$ & $0.5116(5)$ & $0.8243(5)$ & $0.4920(5)$ & $0.4855(5)$ \\
$x_{2}$ & $0.1688(1)$ & $0.4403(1)$ & $0.1043(1)$ & $0.2380(2)$ \\
$x_{3}$ & $0.2505(2)$ & $0.6013(2)$ & $0.3640(2)$ & $0.1217(1)$ \\
$x_{4}$ & $0.3930(3)$ & $0.7437(3)$ & $0.4480(4)$ & $0.2979(3)$ \\
$x_{5}$ & $0.4888(4)$ & $0.7738(4)$ & $0.3698(3)$ & $0.4579(4)$ \\
\hline
\end{tabular}

distance values of the fixed type approach are larger than those of the other approaches due to the PFPIS and PFNIS. The methods of the classical type, the fixed type, and the scalar type employed the proposed distance measurement method; however, the results are partially inconsistent $\left(x_{2}\right.$ has the minimum distance from $x^{+}$with the classical type approach and the fixed type approach, whereas $x_{3}$ has the minimum distance with the scalar type approach) due to the differences in their PFPISs and PFNISs. Moreover, the difference between the scored type approach and the scalar type approach mainly depends on $x_{2}$ and $x_{3}$ with respect to both $D\left(x_{i}, x^{+}\right)$and $D\left(x_{i}, x^{-}\right)$, namely, $x_{2}$ has the minimum distance from the PFPIS and the maximum distance from the PFNIS with the scored type approach, whereas $x_{3}$ does with the scalar type approach. In addition, Zhang and Xu's distance measure [5] with the scored type approach considers three parameters $\left(\mu_{p}, v_{p}, h_{p}\right)$ of PFNs in the distance measure equation, whereas the proposed distance measurement method with the scalar type approach considers five parameters $\left(\mu_{p}, v_{p}, r_{p}, d_{p}, \theta_{p}\right)$ in the distance measure equation, which fully represents the properties of PFNs and is more effective and feasible.

Third, we obtain the results regarding the $R C\left(x_{i}\right)$ and the $\zeta\left(x_{i}\right)$, which are utilized to identify the ranking order results via four approaches, which are listed in Table 11.

Table $10 \mid D\left(x_{i}, x^{-}\right)$results based on four approaches.

\begin{tabular}{lllll}
\hline & I (Rank) & II (Rank) & III (Rank) & IV (Rank) \\
\hline$x_{1}$ & $0.1740(5)$ & $0.6900(4)$ & $0.1538(5)$ & $0.1597(5)$ \\
$x_{2}$ & $0.4978(2)$ & $0.7945(3)$ & $0.5400(1)$ & $0.4924(2)$ \\
$x_{3}$ & $0.5316(1)$ & $0.8636(1)$ & $0.5073(2)$ & $0.5262(1)$ \\
$x_{4}$ & $0.4106(3)$ & $0.8436(2)$ & $0.4838(3)$ & $0.4143(3)$ \\
$x_{5}$ & $0.2827(4)$ & $0.6797(5)$ & $0.3120(4)$ & $0.2774(4)$ \\
\hline
\end{tabular}

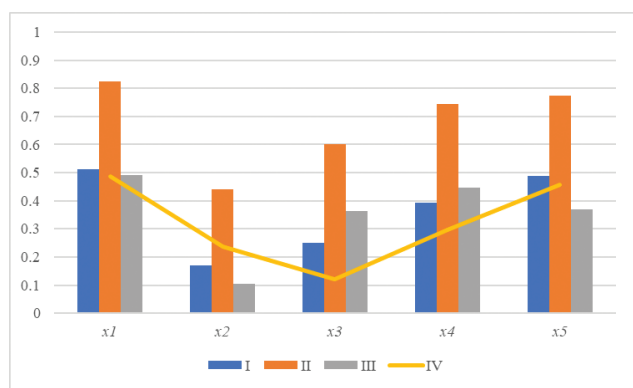

Figure 3 Graphical representation of the $D\left(x_{i}, x^{+}\right)$ results for four approaches.

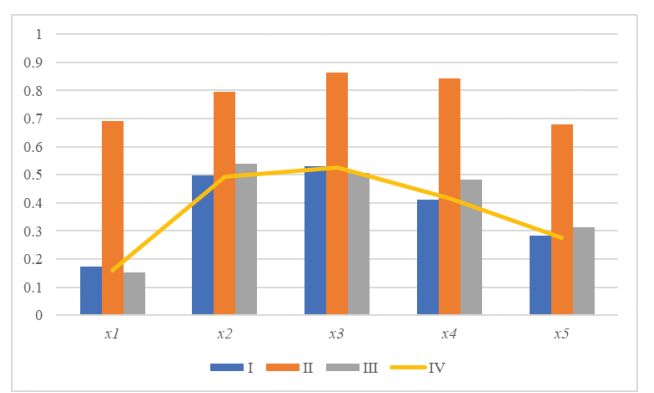

Figure 4 Graphical representation of the $D\left(x_{i}, x^{-}\right)$ results for four approaches. 
To compare the differences of the $R C\left(x_{i}\right)$ and $\zeta\left(x_{i}\right)$ values that were obtained via the four approaches, we plot the results in Fig. 5. The $R C\left(x_{i}\right)$ values from the PF-TOPSIS of the classical type, the fixed type, and the scored type are very close among alternatives and may not differ significantly, whereas the disparity of $R C\left(x_{i}\right)$ from the scalar type approach is more significant among the alternatives. For $\zeta\left(x_{i}\right)$, the results that were obtained with the classical type and the fixed type are closer than those that were obtained with the scored type and the scalar type; hence, the PF-TOPSIS with the scored type and the scalar type can distinguish the alternatives more clearly.

To provide a clearer view of the comparison of the ranking order results that are based on $R C\left(x_{i}\right)$ and $\zeta\left(x_{i}\right)$ from the four approaches, we present Radar charts of the results in Fig. 6. Moreover, we add the ranking order results from Wei and Lu's method [42] (denoted as V) to Fig. 6.

The main differences are those of $x_{2}$ and $x_{3}$ between the scalar type approach and each of the other approaches. The alternative $x_{3}$ is the best alternative that is based on $R C\left(x_{i}\right)$ and $\zeta\left(x_{i}\right)$ from the scalar type approach, which is assigned the second ranking order from the other approaches. The scalar type approach utilizes the scalar function to identify the PFPIS and PFNIS, and the proposed distance measure to calculate the distance from PFPIS and PFNIS, both of which reflects the unique properties of the PFNs, and $x_{3}$ is the best alternative for both $D\left(x_{i}, x^{+}\right)$and $D\left(x_{i}, x^{-}\right)$. Therefore, the results from the scalar type PF-TOPSIS are reasonable. In addition, the result from Wei and Lu' method [42] is the same as that of the scored type approach that is based on $R C\left(x_{i}\right)$ because Wei and Lu's method [42] employs the score function to determine the magnitudes of the

Table $11 \mid R C(x i), \zeta(x i)$ and ranking results from four approaches.

\begin{tabular}{lccrr}
\hline $\boldsymbol{R C}$ & I (Rank) & II (Rank) & III (Rank) & IV Rrank) \\
\hline$x_{1}$ & $0.2538(5)$ & $0.4556(5)$ & $0.2381(5)$ & $0.2475(5)$ \\
$x_{2}$ & $0.7467(1)$ & $0.6434(1)$ & $0.8382(1)$ & $0.6742(2)$ \\
$x_{3}$ & $0.6797(2)$ & $0.5895(2)$ & $0.5822(2)$ & $0.8122(1)$ \\
$x_{4}$ & $0.5110(3)$ & $0.5315(3)$ & $0.5192(3)$ & $0.5817(3)$ \\
$x_{5}$ & $0.3665(4)$ & $0.4676(4)$ & $0.4576(4)$ & $0.3772(4)$ \\
\hline$\zeta$ & I (Rank) & II (Rank) & III (Rank) & IV (Rank) \\
\hline$x_{1}$ & $-2.6807(5)$ & $-1.0036(5)$ & $-4.4163(5)$ & $-1.7367(5)$ \\
$x_{2}$ & $0.0000(1)$ & $0.0000(1)$ & $0.0646(1)$ & $-0.0642(2)$ \\
$x_{3}$ & $-0.4160(2)$ & $-0.278(2)$ & $-2.4916(2)$ & $0.4887(1)$ \\
$x_{4}$ & $-1.5026(3)$ & $-0.627(3)$ & $-3.3437(4)$ & $-0.4644(3)$ \\
$x_{5}$ & $-2.3271(4)$ & $-0.9017(4)$ & $-2.9317(3)$ & $-1.3971(4)$ \\
\hline
\end{tabular}

$\mathrm{RC}$, relative closeness.

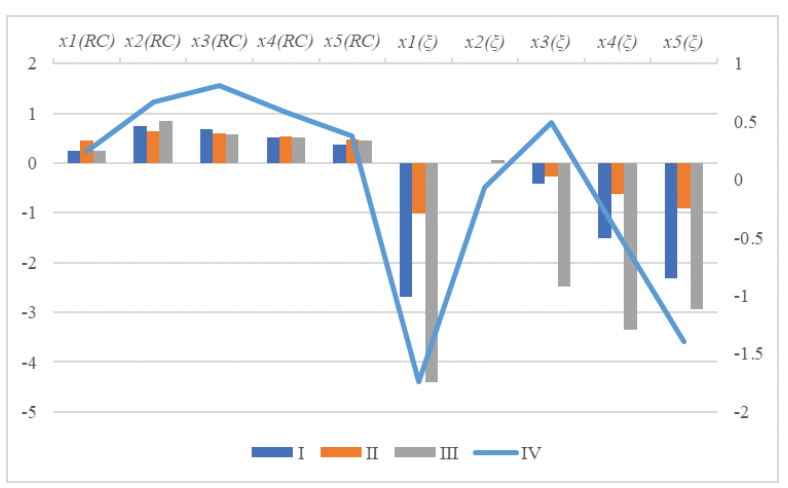

Figure 5 Graphical representation of the ranking orders of five alternatives from four approaches.
PFNs and the distance measure, while ignoring key properties of PFNs, such as the direction of commitment, the strength of commitment, and the radian.

Moreover, we consider the weight that corresponds to the importance of the criterion, which is determined by the decision maker's preference and may influence the ranking result. To evaluate the stability, we assume that all criterion weights are equal. Then, the weight vector of the four criteria in the example in Subsection 5.1 is expressed as $\omega=(0.25,0.25,0.25,0.25)^{T}$. The $R C\left(x_{i}\right)$ and $\zeta\left(x_{i}\right)$ under the same weights that are obtained via the four approaches are listed in Table 12.

Among the alternatives, $R C\left(x_{i}\right)$ and $\zeta\left(x_{i}\right)$ are larger under the same weights. To facilitate comparison, we collect all the ranking order results from the four approaches in Table 13.

According to the results in Tables 12 and 13, the ranking order results that are based on both $R C\left(x_{i}\right)$ and $\zeta\left(x_{i}\right)$ from the scalar type approach are consistent and satisfy the previous weight consideration condition, which demonstrates the stability of the this approach. Comparing the results with the classical type and fixed type approaches, the differences are similar to the previous weight consideration condition. All the approaches are stable except the scored type approach; hence, the PFPIS, the PFNIS, and the distance measure formulas influence $R C\left(x_{i}\right)$ and $\zeta\left(x_{i}\right)$ as well as their corresponding ranking orders of the alternatives.

In line with the properties of the PFNs, the scalar type PF-TOPSIS approach utilizes the scalar function to identify the PFPIS and PFNIS. Moreover, the proposed distance measure can obtain the distances from PFPIS and PFNIS, which were demonstrated to be
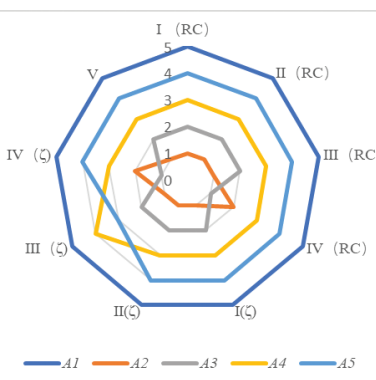

Figure 6 Comparison of the ranking order results based on indices from four approaches.

Table $12 R C(x i), \zeta(x i)$ and their corresponding ranking results from four approaches under the same weights.

\begin{tabular}{ccrrr}
\hline $\boldsymbol{R C}$ & I (Rank) & II (Rank) & III (Rank) & IV (Rank) \\
\hline$x_{1}$ & $0.2468(5)$ & $0.4580(5)$ & $0.2349(5)$ & $0.2558(5)$ \\
$x_{2}$ & $0.7292(1)$ & $0.6355(1)$ & $0.7932(1)$ & $0.6643(2)$ \\
$x_{3}$ & $0.6931(2)$ & $0.5921(2)$ & $0.6204(2)$ & $0.8219(1)$ \\
$x_{4}$ & $0.5382(3)$ & $0.5363(3)$ & $0.5548(3)$ & $0.5992(3)$ \\
$x_{5}$ & $0.3935(4)$ & $0.4776(4)$ & $0.4990(4)$ & $0.4094(4)$ \\
\hline$\zeta$ & I (Rank) & II (Rank) & III (Rank) & IV (Rank) \\
\hline$x_{1}$ & $-2.4847(5)$ & $-0.9238(5)$ & $-3.2283(5)$ & $-1.6707(5)$ \\
$x_{2}$ & $0.0000(1)$ & $0.0000(1)$ & $0.0538(1)$ & $-0.0959(2)$ \\
$x_{3}$ & $-0.2127(2)$ & $-0.2193(2)$ & $-1.2268(2)$ & $0.5257(1)$ \\
$x_{4}$ & $-1.1797(3)$ & $-0.5427(3)$ & $-1.8979(4)$ & $-0.3777(3)$ \\
$x_{5}$ & $-1.9569(4)$ & $-0.7963(4)$ & $-1.8047(3)$ & $-1.2125(4)$ \\
\hline
\end{tabular}

$\mathrm{RC}$, relative closeness. 
Table 13 Ranking order results from four approaches based on the same weights.

\begin{tabular}{cc}
\hline \multicolumn{1}{c}{ Index } & Ranking Order \\
\hline$R C\left(x_{i}\right)$ from I & $x_{2}>x_{3}>x_{4}>x_{5}>x_{1}$ \\
$R C\left(x_{i}\right)$ from II & $x_{2}>x_{3}>x_{4}>x_{5}>x_{1}$ \\
$R C\left(x_{i}\right)$ from III & $x_{2}>x_{3}>x_{4}>x_{5}>x_{1}$ \\
$R C\left(x_{i}\right)$ from IV & $x_{3}>x_{2}>x_{4}>x_{5}>x_{1}$ \\
$\zeta\left(x_{i}\right)$ from I & $x_{2}>x_{3}>x_{4}>x_{5}>x_{1}$ \\
$\zeta\left(x_{i}\right)$ from II & $x_{2}>x_{3}>x_{4}>x_{5}>x_{1}$ \\
$\zeta\left(x_{i}\right)$ from III & $x_{2}>x_{3}>x_{5}>x_{4}>x_{1}$ \\
$\zeta\left(x_{i}\right)$ from IV & $x_{3}>x_{2}>x_{4}>x_{5}>x_{1}$ \\
\hline
\end{tabular}

$\mathrm{RC}$, relative closeness.

relatively stable and accurate for MCDM problems compared to the classical type, the fixed type, and the scored type PF-TOPSIS approaches.

\section{CONCLUSIONS AND FUTURE RESEARCH}

Distance measurement is an essential method for distinguishing the objects in fuzzy environment. Considering the properties of PFNs, we proposed a novel distance measure that includes five parameters: the membership degree, the nonmembership degree, the strength of commitment, the direction of commitment, and the radian. This measure calculates the distance between two PFNs more accurately. Then, we applied the novel distance measurement method in TOPSIS with PF information to solve MCDM problems. On an example, we demonstrate that the scalar type PF-TOPSIS approach is effective and accurate.

The main contributions of this paper are summarized as follows: (1) This paper proposed a novel distance measurement method that is based on the properties of the PF sets, considering the parameters of $\mu_{p}, v_{p}, r_{p}, d_{p}$, and $\theta_{p}$, employing the absolute squared deviations of $\mu_{p}, v_{p}$, and $r_{p}$, and using the absolute deviations of $d_{p}$ and $\sin \left(\theta_{p}\right)$. The proposed distance measure reflects both the length distance and the angular distance, which ensures that the space of the PF sets is larger than that of the IF sets. The proposed distance measure satisfies the useful properties in the proven theorems. Moreover, it is consistent with the deviations of magnitude values. The proposed distance measure effectively overcame the maximum distance problem using the measures by Li and Zeng [32] and Zeng et al. [33], the transitivity problem using the measures by Zhang and $\mathrm{Xu}$ [5], and the great disparities problem using the measures by Li and Zeng [32] and Zeng et al. [33] (2) This paper improved the PF-TOPSIS methodology substantial. For the PFPIS and the PFNIS, we compared the existing magnitude comparison methods and selected the scalar function that fully reflects the characteristics of the PFNs. Then, we applied the novel distance measurement method to calculate the distances from the PFPIS and the PFNIS, which has been demonstrated to be more effective and accurate. (3) This paper proposed the more practical and precise PF-TOPSIS approach for addressing MCDM problems. Due to the full consideration of the properties and characteristics of PF sets, the scalar type PF-TOPSIS approach could handle the MCDM problems more effectively and precisely under the PF environment compared to the classical type, the fixed type, and the scored type PF-TOPSIS approaches.
This study utilized $\sin \left(\theta_{p}\right)$ to represent the radian of commitment; however, we haven't determined whether $\cos \left(\theta_{p}\right)$ serves the same function as $\sin \left(\theta_{p}\right)$ in distance measure for PFNs. Moreover, we haven't applied the Euclidean distance measure or the generalized distance measure to PF-TOPSIS for MCDM problems. In the future, we can draw on the experience of the distance measure of IF sets by quaternary functions [30] to explore new properties of PFNs. We should compare the differences between $\cos \left(\theta_{p}\right)$ and $\sin \left(\theta_{p}\right)$ in distance measures for PFNs, then apply the other two distance measurement methods in PF-TOPSIS to evaluate their effectiveness and feasibility for MCDM problems.

\section{DATA AVAILABILITY STATEMENT}

The datasets that were generated during and/or analyzed during the current study are available from the corresponding author on reasonable request.

\section{CONFLICT OF INTEREST}

The authors declare that there is no conflict of interest regarding the publication of this paper.

\section{ETHICAL APPROVAL}

This article does not contain any studies with human participants or animals that were performed by the authors.

\section{Funding Statement}

The corresponding author Ting-Yu Chen is grateful for grant funding support from the Taiwan Ministry of Science and Technology (MOST 108-2410-H-182-014-MY2) and Chang Gung Memorial Hospital (BMRP 574 and CMRPD2F0203) during the completion of this study.

\section{ACKNOWLEDGMENTS}

The authors acknowledge the assistance of the respected editor and the anonymous referees via their insightful and constructive comments, which helped improve the overall quality of the paper. The corresponding author Ting-Yu Chen is grateful for grant funding support from the Taiwan Ministry of Science and Technology (MOST 108-2410-H-182-014-MY2) and Chang Gung Memorial Hospital (BMRP 574 and CMRPD2F0203) during the completion of this study.

\section{REFERENCES}

[1] L.A. Zadeh, Fuzzy sets, Info. Control. 8 (1965), 338-353.

[2] K.T. Atanassov, Intuitionistic fuzzy sets, Fuzzy Sets Syst. 20 (1986), $87-96$.

[3] R.R. Yager, Pythagorean fuzzy subsets, in Proceedings of Joint IFSA World Congress and NAFIPS Annual Meeting, Edmonton, 2013, pp. 57-61.

[4] R.R. Yager, Pythagorean membership grades in multi-criteria decision making, IEEE Trans. Fuzzy Syst. 22 (2014), 958-965. 
[5] X.L. Zhang, Z.S. Xu, Extension of TOPSIS to multiple criteria decision making with Pythagorean fuzzy sets, Int. J. Intell. Syst. 29 (2014), 1061-1078.

[6] S.Z. Zeng, J.P. Chen, X.S. Li, A hybrid method for Pythagorean fuzzy multiple-criteria decision making, Int. J. Info. Technol. Decis. Making. 15 (2016), 403-422.

[7] H. Gary, A new improved score function of an interval-valued Pythagorean fuzzy set based TOPSIS method, Int. J. Uncertainty Quantification. 7 (2017), 463-474.

[8] A. Biswas, B. Sarkar, Pythagorean fuzzy TOPSIS for multicriteria group decision-making with unknow weight information through entropy measure, Int. J. Intell. Syst. 34 (2019), 1108-1128.

[9] C.X. Yu, Y.F. Shao, K. Wang, L.P. Zhang, A group decision making sustainable supplier selection approach using extended TOPSIS under interval-valued Pythagorean fuzzy environment, Expert Syst. Appl. 121 (2019), 1-17.

[10] M. Akram, W. Dudek, F. Ilyas, Group decision-making based on Pythagorean fuzzy TOPSIS method, Int. J. Intell. Syst. 34 (2019), $1455-1475$.

[11] P.J. Ren, Z.S. Xu, X.J. Gou, Pythagorean fuzzy TODIM approach to multi-criteria decision making. Appl. Soft Comput. 42 (2016), 246-259.

[12] A. Biswas, B. Sarkar, Interval-valued Pythagorean fuzzy TODIM approach through point operator-based similarity measures for multicriteria group decision making, Kybernetes. 48 (2019), 496-519.

[13] X.L. Zhang, Multicriteria Pythagorean fuzzy decision analysis: A hierarchical QUALIFLEX approach with the closeness indexbased ranking, Info. Sci. 330 (2016), 104-124.

[14] T.Y. Chen, Remoteness index-based Pythagorean fuzzy VIKOR methods with a generalized distance for multiple criteria decision analysis. Info. Fusion. 41 (2017), 129-150.

[15] M. Gul, M.F. Ak, A.F. Guneri, Pythagorean fuzzy VIKOR-based approach for safety risk assessment in mine industry, J. Safety Res. 69 (2019), 135-153.

[16] D. Liang, Y. Zhang, Z. Xu, A. Jamaldeen, Pythagorean fuzzy VIKOR approaches based on TODIM for evaluating internet banking website quality of Ghanaian banking industry, Appl. Soft Comput. 78 (2019), 583-594.

[17] J.C. Wang, T.Y. Chen, Multiple criteria decision analysis using correlation-based precedence indices within Pythagorean fuzzy uncertain environments, Int. J. Comput. Intell. Syst. 11 (2018), 911-924.

[18] E. Haktanır, C. Kahraman, A novel interval-valued Pythagorean fuzzy QFD method and its application to solar photovoltaic technology development, Comput. Ind. Eng. 132 (2019), 361-372.

[19] H.C. Liu, M.Y. Quan, H. Shi, C. Guo, An integrated MCDM method for robot selection under interval-valued Pythagorean uncertain linguistic environment, Int. J. Intell. Syst. 34 (2018), $188-214$.

[20] Y. Yang, Z.S. Chen, Y.H. Chen, K.S. Chin, Interval-valued Pythagorean fuzzy Frank power aggregation operators based on an isomorphic Frank dual triple, Int. J. Comput. Intell. Syst. 11 (2018), 1091-1110.

[21] C.L. Hwang, K.S. Yoon, Multiple Attribute Decision Methods and Applications, Springer, Berlin, 1981.

[22] S.P. Wan, Z. Jin, F. Wang, A new ranking method for Pythagorean fuzzy numbers, in 2017 12th International Conference on Intelligent Systems and Knowledge Engineering (ISKE), Nanjing, 2017.
[23] I. Bloch, On fuzzy distances and their use in image process under impression, Pattern Recognit. Lett. 32 (1999), 1873-1895.

[24] X. Li, B.D. Liu, On distance between fuzzy variables, J. Intell. Fuzzy Syst. 19 (2008), 197-204.

[25] H. Bustince, E. Barrenechea, M. Pagola, Relationship between restricted dissimilarity functions, restricted equivalence functions and normal EN-functions: image thresholding invariant, Pattern Recognit. Lett. 29 (2008), 525-536.

[26] Y.F. Li, K.Y. Qin, X.X. He, Dissimilarity functions and divergence measures between fuzzy sets, Info. Sci. 288 (2014), 15-26.

[27] Y.F. Li, K.Y. Qin, X.X. He, Some new approaches to constructing similarity measures, Fuzzy Sets Syst. 234 (2014), 46-60.

[28] W.Q. Wang, X.L. Xin, Distance measure between intuitionistic fuzzy sets, Pattern Recognit. Lett. 26 (2005), 2063-2069.

[29] P. Grzegorzewski, Distance between intuitionistic fuzzy sets and/or interval-valued fuzzy sets based on the Hansdorff metric, Fuzzy Sets Syst. 148 (2004), 319-328.

[30] I. Montes, N.R. Pal, V. Janiš, S. Montes, Divergence measures for intuitionistic fuzzy sets, IEEE Trans. Fuzzy Syst. 23 (2015), 444-456.

[31] X.X. He, Y.F. Li, K.Y. Qin, D. Meng, Distance measures on intuitionistic fuzzy based on intuitionistic fuzzy dissimilarity functions, Soft Comput. (2019).

[32] D.Q. Li, W.Y. Zeng, Distance measure of Pythagorean fuzzy sets, Int. J. Intell. Syst. 33 (2018), 348-361.

[33] W.Y. Zeng, D.Q. Li, Q. Yin, Distance and similarity measures of Pythagorean fuzzy sets and their applications to multiple criteria group decision making, Int. J. Intell. Syst. 33 (2018), 2236-2254.

[34] H.D. Wang, S.F. He, X.H. Pan, A new bi-directional projection model based on Pythagorean uncertain linguistic variable, Information. 9 (2018), 23-34.

[35] L.P. Yu, S.Z. Zeng, J.M. Merigo, C.H. Zhang, A new distance measure based on the weighted induced method and its application to Pythagorean fuzzy multiple attribute group decision making, Int. J. Intell. Syst. 34 (2019), 1440-1454.

[36] X.D. Peng, W.Q. Li, Algorithms for interval-valued Pythagorean fuzzy sets in emergency decision making based on multiparametric similarity measures and WSBA, IEEE Access. 7 (2019), 7419-7441.

[37] X.D. Peng, Y. Yang, Some results for Pythagorean fuzzy sets, Int. J. Intell. Syst. 30 (2015), 1133-1160.

[38] T.Y. Chen, A novel PROMETHEE-based outranking approach for multiple criteria decision analysis with Pythagorean fuzzy information, IEEE Access. 6 (2018), 54495-54506.

[39] E. Szmidt, J. Kacprzyk, Distance between intuitionistic fuzzy sets, Fuzzy Sets Syst. 114 (2000), 505-518.

[40] Q. Wu, W. Lin, L. Zhou, Y. Chen, H. Chen, Enhancing multiple attribute group decision making flexibility based on information fusion technique and hesitant Pythagorean fuzzy sets, Comput. Ind. Eng. 127 (2019), 954-970.

[41] A. Hadi-Vencheh, M. Mirjaberi, Fuzzy inferior ratio method for multiple attribute decision making problems, Inf. Sci. 277 (2014), 263-272.

[42] G.W. Wei, M. Lu, Pythagorean fuzzy power aggregation operators in multiple attribute decision making, Int. J. Intell. Syst. 33 (2018), 169-186. 